\title{
STRATIFIED CRITICAL POINTS ON THE REAL MILNOR FIBRE AND INTEGRAL-GEOMETRIC FORMULAS
}

\author{
NICOLAS DUTERTRE
}

Dedicated to professor David Trotman on his 60th birthday

\begin{abstract}
Let $(X, 0) \subset\left(\mathbb{R}^{n}, 0\right)$ be the germ of a closed subanalytic set and let $f$ and $g:(X, 0) \rightarrow(\mathbb{R}, 0)$ be two subanalytic functions. Under some conditions, we relate the critical points of $g$ on the real Milnor fibre $X \cap f^{-1}(\delta) \cap B_{\epsilon}, 0<|\delta| \ll \epsilon \ll 1$, to the topology of this fibre and other related subanalytic sets. As an application, when $g$ is a generic linear function, we obtain an "asymptotic" Gauss-Bonnet formula for the real Milnor fibre of $f$. From this Gauss-Bonnet formula, we deduce "infinitesimal" linear kinematic formulas.
\end{abstract}

\section{INTRODUCTION}

Let $F=\left(f_{1}, \ldots, f_{k}\right):\left(\mathbb{C}^{n}, 0\right) \rightarrow\left(\mathbb{C}^{k}, 0\right), 2 \leq k \leq n$, be a complete intersection with isolated singularity. The Lê-Greuel formula [21, 22] states that

$$
\mu\left(F^{\prime}\right)+\mu(F)=\operatorname{dim}_{\mathbb{C}} \frac{\mathcal{O}_{\mathbb{C}^{n}, 0}}{I},
$$

where $F^{\prime}:\left(\mathbb{C}^{n}, 0\right) \rightarrow\left(\mathbb{C}^{k-1}, 0\right)$ is the map with components $f_{1}, \ldots, f_{k-1}$, $I$ is the ideal generated by $f_{1}, \ldots, f_{k-1}$ and the $(k \times k)$-minors $\frac{\partial\left(f_{1}, \ldots, f_{k}\right)}{\partial\left(x_{i_{1}}, \ldots, x_{i_{k}}\right)}$ and $\mu(F)$ (resp. $\mu\left(F^{\prime}\right)$ ) is the Milnor number of $F$ (resp $F^{\prime}$ ). Hence the Lê-Greuel formula gives an algebraic characterization of a topological data, namely the sum of two Milnor numbers. However, since the right-hand side of the above equality is equal to the number of critical points of $f_{k}$, counted with multiplicity, on the Milnor fibre of $F^{\prime}$, the Lê-Greuel formula can be also viewed as a topological characterization of this number of critical points.

Many works have been devoted to the search of a real version of the Lê-Greuel formula. Let us recall them briefly. We consider an analytic map-germ $F=\left(f_{1}, \ldots, f_{k}\right):\left(\mathbb{R}^{n}, 0\right) \rightarrow\left(\mathbb{R}^{k}, 0\right), 2 \leq k \leq n$, and we denote by $F^{\prime}$ the map-germ $\left(f_{1}, \ldots, f_{k-1}\right):\left(\mathbb{R}^{n}, 0\right) \rightarrow\left(\mathbb{R}^{k-1}, 0\right)$. Some authors investigated the following difference:

$$
D_{\delta, \delta^{\prime}}=\chi\left(F^{\prime-1}(\delta) \cap\left\{f_{k} \geq \delta^{\prime}\right\} \cap B_{\epsilon}\right)-\chi\left(F^{\prime-1}(\delta) \cap\left\{f_{k} \leq \delta^{\prime}\right\} \cap B_{\epsilon}\right),
$$

where $\left(\delta, \delta^{\prime}\right)$ is a regular value of $F$ such that $0 \leq\left|\delta^{\prime}\right| \ll|\delta| \ll \epsilon$.

In 12 , we proved that

$$
D_{\delta, \delta^{\prime}} \equiv \operatorname{dim}_{\mathbb{R}} \frac{\mathcal{O}_{\mathbb{R}^{n}, 0}}{I} \bmod 2,
$$


where $\mathcal{O}_{\mathbb{R}^{n}, 0}$ is the ring of analytic function-germs at the origin and $I$ is the ideal generated by $f_{1}, \ldots, f_{k-1}$ and all the $k \times k$ minors $\frac{\partial\left(f_{k}, f_{1}, \ldots, f_{k-1}\right)}{\partial\left(x_{i_{1}}, \ldots, x_{i_{k}}\right)}$. This is only a mod 2 relation and we may ask if it is possible to get a more precise relation.

When $k=n$ and $f_{k}=x_{1}^{2}+\cdots+x_{n}^{2}$, according to Aoki et al. ([1], [3] $), D_{\delta, 0}=\chi\left(F^{\prime-1}(\delta) \cap B_{\varepsilon}\right)=2 \operatorname{deg}_{0} H$ and $2 \operatorname{deg}_{0} H$ is the number of semibranches of $F^{\prime-1}(0)$, where

$$
H=\left(\frac{\partial\left(f_{n}, f_{1}, \ldots, f_{n-1}\right)}{\partial\left(x_{1}, \ldots, x_{n}\right)}, f_{1}, \ldots, f_{n-1}\right) .
$$

They proved a similar formula in the case $f_{k}=x_{n}$ in [2] and Szafraniec generalized all these results to any $f_{k}$ in [23].

When $k=2$ and $f_{2}=x_{1}$, Fukui [18] stated that

$$
D_{\delta, 0}=-\operatorname{sign}(-\delta)^{n} \operatorname{deg}_{0} H
$$

where $H=\left(f_{1}, \frac{\partial f_{1}}{\partial x_{2}}, \ldots, \frac{\partial f_{1}}{\partial x_{n}}\right)$. Several generalizations of Fukui's formula are given in [19], [11, [20] and [13].

In all these papers, the general idea is to count algebraically the critical points of a Morse perturbation of $f_{k}$ on $F^{\prime-1}(\delta) \cap B_{\epsilon}$ and to express this sum in two ways: as a difference of Euler characteristics and as a topological degree. Using the Eisenbud-Levine formula [16], this latter degree can be expressed as a signature of a quadratic form and so, we obtain an algebraic expression for $D_{\delta, \delta^{\prime}}$.

In this paper, we give a real and stratified version of the Lê-Greuel formula. We restrict ourselves to the topological aspect and relate a sum of indices of critical points on a real Milnor fibre to some Euler characteristics (this is also the point of view adopted in [7]). More precisely, we consider a germ of a closed subanalytic set $(X, 0) \subset\left(\mathbb{R}^{n}, 0\right)$ and a subanalytic function $f:(X, 0) \rightarrow(\mathbb{R}, 0)$. We assume that $X$ is contained in a open set $U$ of $\mathbb{R}^{n}$ and that $f$ is the restriction to $X$ of a $C^{2}$-subanalytic function $F: U \rightarrow \mathbb{R}$. We denote by $X^{f}$ the set $X \cap f^{-1}(0)$ and we equip $X$ with a Thom stratification adapted to $X^{f}$. If $0<|\delta| \ll \epsilon \ll 1$ then the real Milnor fibre of $f$ is defined by

$$
M_{f}^{\delta, \epsilon}=f^{-1}(\delta) \cap X \cap B_{\epsilon} .
$$

We consider another subanalytic function $g:(X, 0) \rightarrow(\mathbb{R}, 0)$ and we assume that it is the restriction to $X$ of a $C^{2}$-subanalytic function $G: U \rightarrow \mathbb{R}$. We denote by $X^{g}$ the intersection $X \cap g^{-1}(0)$. Under two conditions on $g$, we study the topological behaviour of $g_{\mid M_{f}^{\delta, \epsilon}}$.

We recall that if $Z \subset \mathbb{R}^{n}$ is a closed subanalytic set, equipped with a Whitney stratification and $p \in Z$ is an isolated critical point of a subanalytic function $\phi: Z \rightarrow \mathbb{R}$, restriction to $Z$ of a $C^{2}$-subanalytic function $\Phi$, then the index of $\phi$ at $p$ is defined as follows:

$$
\operatorname{ind}(\phi, Z, p)=1-\chi\left(Z \cap\{\phi=\phi(p)-\eta\} \cap B_{\epsilon}(p)\right),
$$


where $0<\eta \ll \epsilon \ll 1$ and $B_{\epsilon}(p)$ is the closed ball of radius $\epsilon$ centered at $p$. Let $p_{1}^{\delta, \epsilon}, \ldots, p_{r}^{\delta, \epsilon}$ be the critical points of $g$ on $X \cap f^{-1}(\delta) \cap \stackrel{\circ}{B}_{\epsilon}$, where $\stackrel{\circ}{B}_{\epsilon}$ denotes the open ball of radius $\epsilon$. We set

$$
\begin{gathered}
I(\delta, \epsilon, g)=\sum_{i=1}^{r} \operatorname{ind}\left(g, X \cap f^{-1}(\delta), p_{i}^{\delta, \epsilon}\right), \\
I(\delta, \epsilon,-g)=\sum_{i=1}^{r} \operatorname{ind}\left(-g, X \cap f^{-1}(\delta), p_{i}^{\delta, \epsilon}\right) .
\end{gathered}
$$

Our main theorem (Theorem 3.10) is the following:

$I(\delta, \epsilon, g)+I(\delta, \epsilon,-g)=2 \chi\left(M_{f}^{\delta, \epsilon}\right)-\chi\left(X \cap f^{-1}(\delta) \cap S_{\epsilon}\right)-\chi\left(X^{g} \cap f^{-1}(\delta) \cap S_{\epsilon}\right)$.

As a corollary (Corollary 3.11), when $f:(X, 0) \rightarrow(\mathbb{R}, 0)$ has an isolated stratified critical point at 0 , we obtain that

$$
I(\delta, \epsilon, g)+I(\delta, \epsilon,-g)=2 \chi\left(M_{f}^{\delta, \epsilon}\right)-\chi\left(\operatorname{Lk}\left(X^{f}\right)\right)-\chi\left(\operatorname{Lk}\left(X^{f} \cap X^{g}\right)\right),
$$

where $\operatorname{Lk}(-)$ denotes the link at the origin.

Then we apply these results when $g$ is a generic linear form to get an asymptotic Gauss-Bonnet formula for $M_{f}^{\delta, \epsilon}$ (Theorem 4.5). In the last section, we use this asymptotic Gauss-Bonnet formula to prove infinitesimal linear kinematic formulas for closed subanalytic germs (Theorem 5.5), that generalize the Cauchy-Crofton formula for the density due to Comte [8].

The paper is organized as follows. In Section 2, we prove several lemmas about critical points on the link of a subanalytic set. Section 3 contains real stratified versions of the Lê-Greuel formula. In Section 4, we establish the asymptotic Gauss-Bonnet formula and in Section 5, the infinitesimal linear kinematic formulas.

The author is grateful to Vincent Grandjean for a very useful discussion on generic distance functions.

The author is partially supported by the program

"Catédras Lévi-Strauss-USP/French Embassy, no. 2012.1.62.55.7".

This paper was written while the author was visiting the Instituto de Ciências Matemáticas e de Computação, Universidade de São Paulo - Campus de São Carlos. He thanks this institution, especially Raimundo Araújo dos Santos and Nivaldo Grulha, for the hospitality.

\section{LEMmAS ON CRITICAL POINTS ON THE LINK OF A STRATUM}

In this section, we study the behaviour of the critical points of a $C^{2}$ subanalytic function on the link of stratum that contains 0 in its closure, for a generic choice of the distance function to the origin.

Let $Y \subset \mathbb{R}^{n}$ be a $C^{2}$-subanalytic set such that 0 belongs to its closure $\bar{Y}$. Let $\theta: \mathbb{R}^{n} \rightarrow \mathbb{R}$ be a $C^{2}$-subanalytic function such that $\theta(0)=0$. We will first study the behaviour of the critical points of $\theta_{\mid Y}: Y \rightarrow \mathbb{R}$ in 
the neighborhood of 0 , and then the behaviour of the critical points of the restriction of $\theta$ to the link of 0 in $Y$.

Lemma 2.1. The critical points of $\theta_{\mid Y}$ lie in $\{\theta=0\}$ in a neighborhood of 0 .

Proof. By the Curve Selection Lemma, we can assume that there is a $C^{1}$ subanalytic curve $\gamma:[0, \nu[\rightarrow \bar{Y}$ such that $\gamma(0)=0$ and $\gamma(t)$ is a critical point of $\theta_{\mid Y}$ for $\left.t \in\right] 0, \nu[$. Therefore, we have

$$
(\theta \circ \gamma)^{\prime}(t)=\left\langle\nabla \theta_{\mid Y}(\gamma(t)), \gamma^{\prime}(t)\right\rangle=0
$$

since $\gamma^{\prime}(t)$ is tangent to $Y$ at $\gamma(t)$. This implies that $\theta \circ \gamma(t)=\theta \circ \gamma(0)=$ 0 .

Let $\rho: \mathbb{R}^{n} \rightarrow \mathbb{R}$ be another $C^{2}$-subanalytic function such that $\rho^{-1}(a)$ intersects $Y$ transversally. Then the set $Y \cap\{\rho \leq a\}$ is a manifold with boundary. Let $p$ be a critical point of $\theta_{\mid Y \cap\{\rho \leq a\}}$ which lies in $Y \cap\{\rho=a\}$ and which is not a critical point of $\theta_{\mid Y}$. This implies that

$$
\nabla \theta_{\mid Y}(p)=\lambda(p) \nabla \rho_{\mid Y}(p),
$$

with $\lambda(p) \neq 0$.

Definition 2.2. We say that $p \in Y \cap\{\rho=a\}$ is an outwards-pointing (resp. inwards-pointing) critical point of $\theta_{\mid Y \cap\{\rho \leq a\}}$ if $\lambda(p)>0$ (resp. $\lambda(p)<0$ ).

Now let us assume that $\rho: \mathbb{R}^{n} \rightarrow \mathbb{R}$ is a distance function to the origin which means that $\rho \geq 0$ and $\rho^{-1}(0)=\{0\}$ in a neighborhood of 0 . By Lemma 2.1, we know that for $\epsilon>0$ small enough, the level $\rho^{-1}(\epsilon)$ intersects $Y$ transversally. Let $p^{\epsilon}$ be a critical point of $\theta_{\mid Y \cap \rho^{-1}(\epsilon)}$ such that $\theta\left(p^{\epsilon}\right) \neq 0$. This means that there exists $\lambda\left(p^{\epsilon}\right)$ such that

$$
\nabla \theta_{\mid Y}\left(p^{\epsilon}\right)=\lambda\left(p^{\epsilon}\right) \nabla \rho_{\mid Y}\left(p^{\epsilon}\right) .
$$

Note that $\lambda\left(p^{\epsilon}\right) \neq 0$ because $\nabla \theta_{\mid Y}\left(p^{\epsilon}\right) \neq 0$ for $\theta\left(p^{\epsilon}\right) \neq 0$.

Lemma 2.3. The point $p^{\epsilon}$ is an outwards-pointing (resp. inwards-pointing) for $\theta_{\mid Y \cap\{\rho \leq \epsilon\}}$ if and only if $\theta\left(p^{\epsilon}\right)>0$ (resp. $\left.\theta\left(p^{\epsilon}\right)<0\right)$.

Proof. Let us assume that $\lambda\left(p^{\epsilon}\right)>0$. By the Curve Selection Lemma, there exists a $C^{1}$-subanalytic curve $\gamma:\left[0, \nu\left[\rightarrow \bar{Y}\right.\right.$ passing through $p^{\epsilon}$ such that $\gamma(0)=0$ and for $t \neq 0, \gamma(t)$ is a critical point of $\theta_{\mid Y \cap\{\rho=\rho(\gamma(t))\}}$ with $\lambda(\gamma(t))>0$. Therefore we have

$$
(\theta \circ \gamma)^{\prime}(t)=\left\langle\nabla \theta_{\mid Y}(\gamma(t)), \gamma^{\prime}(t)\right\rangle=\lambda(\gamma(t))\left\langle\nabla \rho_{\mid Y}(\gamma(t)), \gamma^{\prime}(t)\right\rangle .
$$

But $(\rho \circ \gamma)^{\prime}>0$ for otherwise $(\rho \circ \gamma)^{\prime} \leq 0$ and $\rho \circ \gamma$ would be decreasing. Since $\rho(\gamma(t))$ tends to 0 as $t$ tends to 0 , this would imply that $\rho \circ \gamma(t) \leq 0$, which is impossible. We can conclude that $(\theta \circ \gamma)^{\prime}>0$ and that $\theta \circ \gamma$ is strictly increasing. Since $\theta \circ \gamma(t)$ tends to 0 as $t$ tends to 0 , we see that $\theta \circ \gamma(t)>0$ for $t>0$. Similarly if $\lambda\left(p^{\epsilon}\right)<0$ then $\theta\left(p^{\epsilon}\right)<0$. 
Now we will study these critical points for a generic choice of the distance function. We denote by $\operatorname{Sym}\left(\mathbb{R}^{n}\right)$ the set of symmetric $n \times n$-matrices with real entries, by $\operatorname{Sym}^{*}\left(\mathbb{R}^{n}\right)$ the open dense subset of such matrices with nonzero determinant and by $\operatorname{Sym}^{*,+}\left(\mathbb{R}^{n}\right)$ the open subset of these invertible matrices that are positive definite or negative definite. Note that these sets are semi-algebraic. For each $A \in \operatorname{Sym}^{*,+}\left(\mathbb{R}^{n}\right)$, we denote by $\rho_{A}$ the following quadratic form:

$$
\rho_{A}(x)=\langle A x, x\rangle \text {. }
$$

We denote by $\Gamma_{\theta, A}^{Y}$ the following subanalytic polar set:

$$
\Gamma_{\theta, A}^{Y}=\left\{x \in Y \mid \operatorname{rank}\left[\nabla \theta_{\mid Y}(x), \nabla \rho_{A \mid Y}(x)\right]<2\right\},
$$

and by $\Sigma_{\theta}^{Y}$ the set of critical points of $\theta_{\mid Y}$. Note that $\Sigma_{\theta}^{Y} \subset\{\theta=0\}$ by Lemma 2.1

Lemma 2.4. For almost all $A$ in $\operatorname{Sym}^{+, *}\left(\mathbb{R}^{n}\right), \Gamma_{\theta, A}^{Y} \backslash\left(\Sigma_{\theta}^{Y} \cup\{0\}\right)$ is a $C^{1}$ subanalytic curve (possible empty) in a neighborhood of 0.

Proof. We can assume that $\operatorname{dim} Y>1$. Let

$$
\begin{aligned}
Z=\left\{(x, A) \in \mathbb{R}^{n} \times \operatorname{Sym}^{+, *}\left(\mathbb{R}^{n}\right) \mid\right. & x \in Y \backslash\left(\Sigma_{\theta}^{Y} \cup\{0\}\right) \\
& \text { and } \left.\operatorname{rank}\left[\nabla \theta_{\mid Y}(x), \nabla \rho_{A \mid Y}(x)\right]<2\right\} .
\end{aligned}
$$

Let $(y, B)$ be a point in $Z$. We can suppose that around $y, Y$ is defined by the vanishing of $k$ subanalytic functions $f_{1}, \ldots, f_{k}$ of class $C^{2}$. Hence in a neighborhood of $(y, B), Z$ is defined be the vanishing of $f_{1}, \ldots, f_{k}$ and the minors

$$
\frac{\partial\left(f_{1}, \ldots, f_{k}, \theta, \rho_{A}\right)}{\partial\left(x_{i_{1}}, \ldots, x_{i_{k+2}}\right)} .
$$

Furthermore, since $y$ does not belong to $\Sigma_{\theta}^{Y}$, we can assume that

$$
\frac{\partial\left(f_{1}, \ldots, f_{k}, \theta\right)}{\partial\left(x_{1}, \ldots, x_{k}, x_{k+1}\right)} \neq 0
$$

in a neighborhood of $y$. Therefore $Z$ is locally defined by $f_{1}=\cdots=f_{k}=0$ and

$$
\frac{\partial\left(f_{1}, \ldots, f_{k}, \theta, \rho_{A}\right)}{\partial\left(x_{1}, \ldots, x_{k+1}, x_{k+2}\right)}=\cdots=\frac{\partial\left(f_{1}, \ldots, f_{k}, \theta, \rho_{A}\right)}{\partial\left(x_{1}, \ldots, x_{k+1}, x_{n}\right)}=0
$$

Let us write $M=\frac{\partial\left(f_{1}, \ldots, f_{k}, \theta\right)}{\partial\left(x_{1}, \ldots, x_{k}, x_{k+1}\right)}$ and for $i \in\{k+2, \ldots, n\}, m_{i}=\frac{\partial\left(f_{1}, \ldots, f_{k}, \theta, \rho_{A}\right)}{\partial\left(x_{1}, \ldots, x_{k+1}, x_{i}\right)}$. If $A=\left[a_{i j}\right]$ then

$$
\rho_{A}(x)=\sum_{i=1}^{n} a_{i i} x_{i}^{2}+2 \sum_{i \neq j} a_{i j} x_{i} x_{j},
$$

and so $\frac{\partial \rho_{A}}{\partial x_{i}}(x)=2 \sum_{j=1}^{n} a_{i j} x_{j}$. For $i \in\{k+1, \ldots, n\}$ and $j \in\{1, \ldots, n\}$, we have

$$
\frac{\partial m_{i}}{\partial a_{i j}}=2 x_{j} M
$$


Since $y \neq 0$, one of the $x_{j}$ 's does not vanish in the neighborhood of $y$ and we can conclude that the rank of

$$
\left[\nabla f_{1}(x), \ldots, \nabla f_{k}(x), \nabla m_{k+2}(x, A), \ldots, \nabla m_{n}(x, A)\right]
$$

is $n-1$ and that $Z$ is a $C^{1}$-subanalytic manifold of dimension $\frac{n(n+1)}{2}+1$. Now let us consider the projection $\pi_{2}: Z \rightarrow \operatorname{Sym}^{+, *}\left(\mathbb{R}^{n}\right),(x, A) \mapsto A$. Bertini-Sard's theorem implies that the set $D_{\pi_{2}}$ of critical values of $\pi_{2}$ is a subanalytic set of dimension strictly less than $\frac{n(n+1)}{2}$. Hence, for all $A \notin D_{\pi_{2}}$, $\pi_{2}^{-1}(A)$ is a $C^{1}$-subanalytic curve (possibly empty). But this set is exactly $\Gamma_{\theta, A}^{Y} \backslash\left(\Sigma_{\theta}^{Y} \cup\{0\}\right)$.

Let $R \subset Y$ be a subanalytic set of dimension strictly less than $\operatorname{dim} Y$. We will need the following lemma.

Lemma 2.5. For almost all $A$ in $\operatorname{Sym}^{+, *}\left(\mathbb{R}^{n}\right), \Gamma_{\theta, A}^{Y} \backslash\left(\Sigma_{\theta}^{Y} \cup\{0\}\right) \cap R$ is a subanalytic set of dimension at most 0 in a neighborhood of 0 .

Proof. Let us put $l=\operatorname{dim} Y$. Since $R$ admits a locally finite subanalytic stratification, we can assume that $R$ is a $C^{2}$-subanalytic manifold of dimension $d$ with $d<l$. Let $W$ be the following subanalytic set:

$$
\begin{aligned}
W=\left\{(x, A) \in \mathbb{R}^{n} \times \operatorname{Sym}^{+, *}\left(\mathbb{R}^{n}\right)\right. & \mid x \in R \backslash\left(\Sigma_{\theta}^{Y} \cup\{0\}\right) \\
& \text { and } \left.\operatorname{rank}\left[\nabla \theta_{\mid Y}(x), \nabla \rho_{A \mid Y}(x)\right]<2\right\} .
\end{aligned}
$$

Using the same method as in the previous lemma, we can prove that $W$ is a $C^{1}$-subanalytic manifold of dimension $\frac{n(n+1)}{2}+1+d-l$ and conclude, remarking that $d-l \leq-1$.

Now we introduce a new $C^{2}$-subanalytic function $\beta: \mathbb{R}^{n} \rightarrow \mathbb{R}$ such that $\beta(0)=0$. We denote by $\Gamma_{\theta, \beta, A}^{Y}$ the following subanalytic polar set:

$$
\Gamma_{\theta, \beta, A}^{Y}=\left\{x \in Y \mid \operatorname{rank}\left[\nabla \theta_{\mid Y}(x), \nabla \beta_{\mid Y}(x), \nabla \rho_{A \mid Y}(x)\right]<3\right\},
$$

and by $\Gamma_{\theta, \beta}^{Y}$ the following subanalytic polar set:

$$
\Gamma_{\theta, \beta}^{Y}=\left\{x \in Y \mid \operatorname{rank}\left[\nabla \theta_{\mid Y}(x), \nabla \beta_{\mid Y}(x)\right]<2\right\} .
$$

Lemma 2.6. For almost all $A$ in $\operatorname{Sym}^{+, *}\left(\mathbb{R}^{n}\right), \Gamma_{\theta, \beta, A}^{Y} \backslash\left(\Gamma_{\theta, \beta}^{Y} \cup\{0\}\right)$ is a $C^{1}$ subanalytic set of dimension at most 2 (possibly empty) in a neighborhood of 0 .

Proof. We can assume that $\operatorname{dim} Y>2$. Let

$$
\begin{array}{r}
Z=\left\{(x, A) \in \mathbb{R}^{n} \times \operatorname{Sym}^{+, *}\left(\mathbb{R}^{n}\right) \mid x \in Y, \operatorname{rank}\left[\nabla \theta_{\mid Y}(x), \nabla \beta_{\mid Y}(x)\right]=2\right. \\
\text { and rank } \left.\left[\nabla \theta_{\mid Y}(x), \nabla \beta_{\mid Y}(x), \nabla \rho_{A \mid Y}(x)\right]<3\right\} .
\end{array}
$$

Let $(y, B)$ be a point in $Z$. We can suppose that around $y, Y$ is defined by the vanishing of $k$ subanalytic functions $f_{1}, \ldots, f_{k}$ of class $C^{2}$. Hence in a 
neighborhood of $(y, B), Z$ is defined by the vanishing of $f_{1}, \ldots, f_{k}$ and the minors

$$
\frac{\partial\left(f_{1}, \ldots, f_{k}, \theta, \beta, \rho_{A}\right)}{\partial\left(x_{i_{1}}, \ldots, x_{i_{k+3}}\right)}
$$

Since $y$ does not belong to $\Gamma_{\theta, \beta}^{Y}$, we can assume that

$$
\frac{\partial\left(f_{1}, \ldots, f_{k}, \theta, \beta\right)}{\partial\left(x_{1}, \ldots, x_{k}, x_{k+1}, x_{k+2}\right)} \neq 0
$$

in a neighborhood of $y$. Therefore $Z$ is locally defined by $f_{1}, \ldots, f_{k}=0$ and

$$
\frac{\partial\left(f_{1}, \ldots, f_{k}, \theta, \beta, \rho_{A}\right)}{\partial\left(x_{1}, \ldots, x_{k+2}, x_{k+3}\right)}=\cdots=\frac{\partial\left(f_{1}, \ldots, f_{k}, \theta, \beta, \rho_{A}\right)}{\partial\left(x_{1}, \ldots, x_{k+2}, x_{n}\right)}=0
$$

It is clear that we can apply the same method as Lemma 2.4 to get the result.

\section{LÊ-GREUEL TYPE FORMULA}

In this section, we prove the Lê-Greuel type formula announced in the introduction.

Let $(X, 0) \subset\left(\mathbb{R}^{n}, 0\right)$ be the germ of a closed subanalytic set and let $f$ : $(X, 0) \rightarrow(\mathbb{R}, 0)$ be a subanalytic function. We assume that $X$ is contained in a open set $U$ of $\mathbb{R}^{n}$ and that $f$ is the restriction to $X$ of a $C^{2}$-subanalytic function $F: U \rightarrow \mathbb{R}$. We denote by $X^{f}$ the set $X \cap f^{-1}(0)$ and by [4], we can equip $X$ with a Thom stratification $\mathcal{V}=\left\{V_{\alpha}\right\}_{\alpha \in A}$ adapted to $X^{f}$. This means that $\left\{V_{\alpha} \in \mathcal{V} \mid V_{\alpha} \nsubseteq X^{f}\right\}$ is a Whitney stratification of $X \backslash X^{f}$ and that for any pair of strata $\left(V_{\alpha}, V_{\beta}\right)$ with $V_{\alpha} \nsubseteq X^{f}$ and $V_{\beta} \subset X^{f}$, the Thom condition is satisfied.

Let us denote by $\Sigma_{\mathcal{V}} f$ the critical locus of $f$. It is the union of the critical loci of $f$ restricted to each stratum, i.e. $\Sigma \mathcal{V} f=\cup_{\alpha} \Sigma\left(f_{\mid V_{\alpha}}\right)$, where $\Sigma\left(f_{\mid V_{\alpha}}\right)$ is the critical set of $f_{\mid V_{\alpha}}: V_{\alpha} \rightarrow \mathbb{R}$. Since $\Sigma_{\mathcal{V}} f \subset f^{-1}(0)$ (see Lemma 2.1), the fibre $f^{-1}(\delta)$ intersects the strata $V_{\alpha}$ 's, $V_{\alpha} \nsubseteq X^{f}$, transversally if $\delta$ is sufficiently small. Hence it is Whitney stratified with the induced stratification $\left\{f^{-1}(\delta) \cap V_{\alpha} \mid V_{\alpha} \nsubseteq X^{f}\right\}$.

By Lemma 2.1, we know that if $\epsilon>0$ is sufficiently small then the sphere $S_{\epsilon}$ intersects $X^{f}$ transversally. By the Thom condition, this implies that there exists $\delta(\epsilon)>0$ such that for each $\delta$ with $0<|\delta| \leq \delta(\epsilon)$, the sphere $S_{\epsilon}$ intersects the fibre $f^{-1}(\delta)$ transversally as well. Hence the set $f^{-1}(\delta) \cap B_{\epsilon}$ is a Whitney stratified set equipped with the following stratification:

$$
\left\{f^{-1}(\delta) \cap V_{\alpha} \cap \stackrel{\circ}{\epsilon}_{\epsilon}, f^{-1}(\delta) \cap V_{\alpha} \cap S_{\epsilon} \mid V_{\alpha} \nsubseteq X^{f}\right\} .
$$

Definition 3.1. We call the set $f^{-1}(\delta) \cap X \cap B_{\epsilon}$, where $0<|\delta| \ll \epsilon \ll 1$, a real Milnor fibre of $f$.

We will use the following notation: $M_{f}^{\delta, \epsilon}=f^{-1}(\delta) \cap X \cap B_{\epsilon}$.

Now we consider another subanalytic function $g:(X, 0) \rightarrow(\mathbb{R}, 0)$ and we assume that it is the restriction to $X$ of a $C^{2}$-subanalytic function $G: U \rightarrow$ 
$\mathbb{R}$. We denote by $X^{g}$ the intersection $X \cap g^{-1}(0)$. Under some restrictions on $g$, we will study the topological behaviour of $g_{\mid M_{f}^{\delta, \epsilon}}$.

First we assume that $g$ satisfies the following Condition (A):

- Condition $(\mathrm{A}): g:(X, 0) \rightarrow(\mathbb{R}, 0)$ has an isolated stratified critical point at 0 .

This means that for each strata $V_{\alpha}$ of $\mathcal{V}, g: V_{\alpha} \backslash\{0\} \rightarrow \mathbb{R}$ is a submersion in a neighborhood of the origin.

In order to give the second assumption on $g$, we need to introduce some polar sets. Let $V_{\alpha}$ be a stratum of $\mathcal{V}$ not contained in $X^{f}$. Let $\Gamma_{f, g}^{V_{\alpha}}$ be the following set:

$$
\Gamma_{f, g}^{V_{\alpha}}=\left\{x \in V_{\alpha} \mid \operatorname{rank}\left[\nabla f_{\mid V_{\alpha}}(x), \nabla g_{\mid V_{\alpha}}(x)\right]<2\right\},
$$

and let $\Gamma_{f, g}$ be the union $\cup \Gamma_{f, g}^{V_{\alpha}}$ where $V_{\alpha} \nsubseteq X^{f}$. We call $\Gamma_{f, g}$ the relative polar set of $f$ and $g$ with respect to the stratification $\mathcal{V}$. We will assume that $g$ satifies the following Condition (B):

- Condition (B): the relative polar set $\Gamma_{f, g}$ is a 1-dimensional $C^{1}$ subanalytic set (possibly empty) in a neighborhood of the origin.

Note that Condition (B) implies that $\overline{\Gamma_{f, g}} \cap X^{f} \subset\{0\}$ in a neighborhood of the origin because the frontiers of the $\Gamma_{f, g}^{V_{\alpha}}$ 's are 0-dimensional.

From Condition (A) and Condition (B), we can deduce the following result.

Lemma 3.2. We have $\overline{\Gamma_{f, g}} \cap X^{g} \subset\{0\}$ in a neighborhood of the origin.

Proof. If it is not the case then there is a $C^{1}$-subanalytic curve $\gamma:[0, \nu[\rightarrow$ $\Gamma_{f, g} \cap X^{g}$ such that $\gamma(0)=0$ and $\gamma(] 0, \nu[) \subset X^{g} \backslash\{0\}$. We can also assume that $\gamma(] 0, \nu[)$ is contained in a stratum $V$. For $t \in] 0, \nu[$, we have

$$
0=(g \circ \gamma)^{\prime}(t)=\left\langle\nabla g_{\mid V}(\gamma(t)), \gamma^{\prime}(t)\right\rangle .
$$

Since $\gamma(t)$ belongs to $\Gamma_{f, g}$ and $\nabla g_{\mid V}(\gamma(t))$ does not vanish for $g:(X, 0) \rightarrow$ $(\mathbb{R}, 0)$ has an isolated stratified critical point at 0 , we can conclude that $\left\langle\nabla f_{\mid V}(\gamma(t)), \gamma^{\prime}(t)\right\rangle=0$ and that $(f \circ \gamma)^{\prime}(t)=0$ for all $\left.t \in\right] 0, \nu[$. Therefore $f \circ \gamma \equiv 0$ because $f(0)=0$ and $\gamma\left(\left[0, \nu[)\right.\right.$ is included in $X^{f}$. This is impossible by the above remark.

Let $\mathcal{B}_{1}, \ldots, \mathcal{B}_{l}$ be the connected components of $\Gamma_{f, g}$, i.e. $\Gamma_{f, g}=\sqcup_{i=1}^{l} \mathcal{B}_{i}$. Each $\mathcal{B}_{i}$ is a $C^{1}$-subanalytic curve along which $f$ is strictly increasing or decreasing and the intersection points of the $\mathcal{B}_{i}$ 's with the fibre $M_{f}^{\delta, \epsilon}$ are exactly the critical points (in the stratified sense) of $g$ on $X \cap f^{-1}(\delta) \cap \stackrel{\circ}{\epsilon}_{\epsilon}$. Let us write

$$
M_{f}^{\delta, \epsilon} \cap \sqcup_{i=1}^{l} \mathcal{B}_{i}=\left\{p_{1}^{\delta, \epsilon}, \ldots, p_{r}^{\delta, \epsilon}\right\}
$$

Note that $r \leq l$.

Let us recall now the definition of the index of an isolated stratified critical point. 
Definition 3.3. Let $Z \subset \mathbb{R}^{n}$ be a closed subanalytic set, equipped with a Whitney stratification. Let $p \in Z$ be an isolated critical point of a subanalytic function $\phi: Z \rightarrow \mathbb{R}$, which is the restriction to $Z$ of a $C^{2}$-subanalytic function $\Phi$. We define the index of $\phi$ at $p$ as follows :

$$
\operatorname{ind}(\phi, Z, p)=1-\chi\left(Z \cap\{\phi=\phi(p)-\eta\} \cap B_{\epsilon}(p)\right),
$$

where $0<\eta \ll \epsilon \ll 1$ and $B_{\epsilon}(p)$ is the closed ball of radius $\epsilon$ centered at $p$.

Our aim is to give a topological interpretation to the following sum:

$$
\sum_{i=1}^{r} \operatorname{ind}\left(g, X \cap f^{-1}(\delta), p_{i}^{\delta, \epsilon}\right)+\operatorname{ind}\left(-g, X \cap f^{-1}(\delta), p_{i}^{\delta, \epsilon}\right) .
$$

For this, we will apply stratified Morse theory to $g_{\mid M_{f}^{\delta, \epsilon}}$. Note that the points $p_{i}$ 's are not the only critical points of $g_{\mid M_{f}^{\delta, \epsilon}}$ and other critical points can occur on the "boundary" $M_{f}^{\delta, \epsilon} \cap S_{\epsilon}$.

The next step is to study the behaviour of these "boundary" critical points for a generic choice of the distance function to the origin. Let $\rho: \mathbb{R}^{n} \rightarrow \mathbb{R}$ be a $C^{2}$-subanalytic function which is a distance function to the origin. We denote by $\tilde{S}_{\epsilon}$ the level $\rho^{-1}(\epsilon)$ and by $\tilde{B}_{\epsilon}$ the set $\{\rho \leq \epsilon\}$. We will focus on the critical points of $g_{\mid X^{f} \cap \tilde{S}_{\epsilon}}$ and $g_{\mid X \cap f^{-1}(\delta) \cap \tilde{S}_{\epsilon}}$, with $0<|\delta| \ll \epsilon \ll 1$.

For each stratum $V$ of $X^{f}$, let

$$
\Gamma_{g, \rho}^{V}=\left\{x \in V \mid \operatorname{rank}\left[\nabla g_{\mid V}(x), \nabla \rho_{\mid V}(x)\right]<2\right\},
$$

and let $\Gamma_{g, \rho}^{X^{f}}=\cup_{V \subset X^{f}} \Gamma_{g, \rho}^{V}$. By Lemma 2.4 and the fact that $g:\left(X^{f}, 0\right) \rightarrow$ $(\mathbb{R}, 0)$ has an isolated stratified critical point at 0 , we can assume that $\Gamma_{g, \rho}^{X^{f}}$ is a $C^{1}$-subanalytic curve in a neighborhood of the origin.

Lemma 3.4. We have $\Gamma_{g, \rho}^{X^{f}} \cap X^{g} \subset\{0\}$ in a neighborhood of the origin.

Proof. Same proof as Lemma 3.2 .

Therefore if $\epsilon>0$ is small enough, $g_{\mid \tilde{S}_{\epsilon} \cap X^{f}}$ has a finite number of critical points. They do not lie in the level $\{g=0\}$ so by Lemma 2.3, they are outwards-pointing for $g_{\mid X^{f} \cap \tilde{B}_{\epsilon}}$ if they lie in $\{g>0\}$ and inwards-pointing if they lie in $\{g<0\}$.

Let us study now the critical points of $g_{\mid X \cap f^{-1}(\delta) \cap \tilde{S}_{\epsilon}}$. We will need the following lemma.

Lemma 3.5. For every $\epsilon>0$ sufficiently small, there exists $\delta(\epsilon)>0$ such that for $0<|\delta| \leq \delta(\epsilon)$, the points $p_{i}^{\delta, \epsilon}$ lie in $\tilde{B}_{\epsilon / 4}$.

Proof. Let

$$
W=\left\{(x, r, y) \in U \times \mathbb{R} \times \mathbb{R} \mid \rho(x)=r, y=f(x) \text { and } x \in \overline{\Gamma_{f, g}}\right\} .
$$


Then $W$ is a subanalytic set of $\mathbb{R}^{n} \times \mathbb{R} \times \mathbb{R}$ and since it is a graph over $\overline{\Gamma_{f, g}}$, its dimension is less or equal to 1 . Let

$$
\begin{aligned}
\pi: \mathbb{R}^{n} \times \mathbb{R} \times \mathbb{R} & \rightarrow \mathbb{R} \times \mathbb{R} \\
(x, r, y) & \mapsto(r, y),
\end{aligned}
$$

be the projection on the last two factors. Then $\pi_{\mid W}: W \rightarrow \pi(W)$ is proper and $\pi(W)$ is a closed subanalytic set in a neighborhood of the origin.

Let us write $Y_{1}=\mathbb{R} \times\{0\}$ and let $Y_{2}$ be the closure of $\pi(W) \backslash Y_{1}$. Since $Y_{2}$ is a curve for $W$ is a curve, 0 is isolated in $Y_{1} \cap Y_{2}$. By Lojasiewicz's inequality, there exists a constant $C>0$ and an integer $N>0$ such that $|y| \geq C r^{N}$ for $(r, y)$ in $Y_{2}$ sufficiently close to the origin. So if $x \in \Gamma_{f, g}$ then $|f(x)| \geq C \rho(x)^{N}$ if $\rho(x)$ is small enough.

Let us fix $\epsilon>0$ small. If $0<|\delta| \leq \frac{1}{C}\left(\frac{\epsilon}{4}\right)^{N}$ and $x \in f^{-1}(\delta) \cap \Gamma_{f, g}$ then $\rho(x) \leq \frac{\epsilon}{4}$

For each stratum $V \nsubseteq X^{f}$, let

$$
\Gamma_{f, g, \rho}^{V}=\left\{x \in V \mid \operatorname{rank}\left[\nabla f_{\mid V}(x), \nabla g_{\mid V}(x), \nabla \rho_{\mid V}(x)\right]<3\right\},
$$

and let $\Gamma_{f, g, \rho}=\cup_{V \nsubseteq X_{f}} \Gamma_{f, g, \rho}^{V}$. By Lemma2.6, we can assume that $\Gamma_{f, g, \rho} \backslash \Gamma_{f, g}$ is a $C^{1}$-subanalytic manifold of dimension 2. Let us choose $\epsilon>0$ small enough so that $\tilde{S}_{\epsilon}$ intersects $\Gamma_{f, g, \rho} \backslash \Gamma_{f, g}$ transversally. Therefore $\left(\Gamma_{f, g, \rho} \backslash\right.$ $\left.\Gamma_{f, g}\right) \cap \tilde{S}_{\epsilon}$ is subanalytic curve. By Lemma 3.4, we can find $\delta(\epsilon)>0$ such that $f^{-1}([\delta(\epsilon),-\delta(\epsilon)]) \cap \tilde{S}_{\epsilon} \cap \Gamma_{f, g}$ is empty and so

$$
f^{-1}([-\delta(\epsilon), \delta(\epsilon)]) \cap\left(\Gamma_{f, g, \rho} \backslash \Gamma_{f, g}\right) \cap \tilde{S}_{\epsilon}=f^{-1}([-\delta(\epsilon), \delta(\epsilon)]) \cap \Gamma_{f, g, \rho} \cap \tilde{S}_{\epsilon} .
$$

Let $C_{1}, \ldots, C_{t}$ be the connected components of $f^{-1}([-\delta(\epsilon), \delta(\epsilon)]) \cap \Gamma_{f, g, \rho} \cap \tilde{S}_{\epsilon}$ whose closure intersects $X^{f} \cap \tilde{S}_{\epsilon}$. Note that by Thom's $\left(a_{f}\right)$-condition, for each $i \in\{1, \ldots, t\}, \overline{C_{i}} \cap X^{f}$ is subset of $\Gamma_{g, \rho}^{X^{f}}$. Let $z_{i}$ be a point in $\overline{C_{i}} \cap X^{f}$. Since $C_{i} \cap X^{f}=\emptyset$, there exists $0<\delta_{i}^{\prime}(\epsilon) \leq \delta(\epsilon)$ such that the fibre $f^{-1}(\delta)$, $0<|\delta| \leq \delta_{i}^{\prime}(\epsilon)$, intersects $C_{i}$ transversally in a neighborhood of $z_{i}$.

Let us choose $\delta$ such that $0<|\delta| \leq \operatorname{Min}\left\{\delta_{i}^{\prime}(\epsilon) \mid i=1, \ldots, t\right\}$. Then the fibre $f^{-1}(\delta)$ intersect the $C_{i}$ 's transversally and $f^{-1}(\delta) \cap\left(\cup_{i} C_{i}\right)$ is exactly the set of critical points of $g_{\mid f^{-1}(\delta) \cap X \cap \tilde{S}_{\epsilon}}$. We have proved:

Lemma 3.6. For $0<|\delta| \ll \epsilon \ll 1, g_{\mid f^{-1}(\delta) \cap X \cap \tilde{S_{\epsilon}}}$ has a finite number of critical points, which are exactly the points in $\Gamma_{f, g, \rho} \cap \tilde{S}_{\epsilon} \cap f^{-1}(\delta)$.

Let $\left\{s_{1}^{\delta, \epsilon}, \ldots, s_{u}^{\delta, \epsilon}\right\}$ be the set of critical points of $g_{\mid f^{-1}(\delta) \cap X \cap \tilde{S_{\epsilon}}}$.

Lemma 3.7. For $i \in\{1, \ldots, u\}, g\left(s_{i}^{\delta, \epsilon}\right) \neq 0$ and $s_{i}^{\delta, \epsilon}$ is outwards-pointing (resp. inwards-pointing) if and only if $g\left(s_{i}^{\delta, \epsilon}\right)>0$ (resp. $\left.g\left(s_{i}^{\delta, \epsilon}\right)<0\right)$.

Proof. Note that $s_{i}^{\delta, \epsilon}$ is necessarily outwards-pointing or inwards-pointing because $s_{i}^{\delta, \epsilon} \notin \Gamma_{f, g}$. 
Assume that for each $\delta>0$ small enough, there exists a point $s_{i}^{\delta, \epsilon}$ such that $g\left(s_{i}^{\delta, \epsilon}\right)=0$. Then we can construct a sequence of points $\left(\sigma_{n}\right)_{n \in \mathbb{N}}$ such that $g\left(\sigma_{n}\right)=0$ and $\sigma_{n}$ is a critical point of $g_{\mid f^{-1}\left(\frac{1}{n}\right) \cap X \cap \tilde{S}_{\epsilon}}$. We can also assume that the points $\sigma_{n}$ 's belong to the same stratum $S$ and that they tend to $\sigma \in V$ where $V \subseteq X^{f}$ and $V \subset \partial \bar{S}$. Therefore we have a decomposition:

$$
\nabla g_{\mid S}\left(\sigma_{n}\right)=\lambda_{n} \nabla f_{\mid S}\left(\sigma_{n}\right)+\mu_{n} \nabla \rho_{\mid S}\left(\sigma_{n}\right) .
$$

Now by Whitney's condition (a), $T_{\sigma_{n}} S$ tends to a linear space $T$ such that $T_{\sigma} V \subset T$. So $\nabla g_{\mid S}\left(\sigma_{n}\right)$ tends to a vector in $T$ whose orthogonal projection on $T_{\sigma} V$ is exactly $\nabla g_{\mid V}(\sigma)$. Similarly $\nabla \rho_{\mid S}\left(\sigma_{n}\right)$ tends to a vector in $T$ whose orthogonal projection on $T_{\sigma} V$ is exactly $\nabla \rho_{\mid V}(\sigma)$. By Thom's condition, $\nabla f_{\mid S}\left(\sigma_{n}\right)$ tends to a vector in $T$ which is orthogonal to $T_{\sigma} V$, so we see that $\nabla g_{\mid V}(\sigma)$ and $\nabla \rho_{\mid V}(\sigma)$ are colinear which means that $\sigma$ is a critical point of $g_{\mid X f \cap \tilde{S}_{\epsilon}}$. But since $g\left(\sigma_{n}\right)=0$, we find that $g(\sigma)=0$, which is impossible by Lemma 3.4. This proves the first assertion.

To prove the second one, we use the same method. Assume that for each $\delta>0$ small enough, there exists a point $s_{i}^{\delta, \epsilon}$ such that $g\left(s_{i}^{\delta, \epsilon}\right)>0$ and $s_{i}^{\delta, \epsilon}$ is an inwards-pointing critical point for $g_{\mid X \cap f^{-1}(\delta) \cap \tilde{S}_{\epsilon}}$. Then we can construct a sequence of points $\left(\tau_{n}\right)_{n \in \mathbb{N}}$ such that $g\left(\tau_{n}\right)>0$ and $\tau_{n}$ is an inwards-pointing critical point for $g_{\mid f^{-1}\left(\frac{1}{n}\right) \cap X \cap \tilde{S}_{\epsilon}}$. We can also assume that the points $\tau_{n}$ 's belong to the same stratum $S$ and that they tend to $\tau \in V$ where $V \subseteq X^{f}$ and $V \subset \partial \bar{S}$. Therefore, we have a decomposition:

$$
\nabla g_{\mid S}\left(\tau_{n}\right)=\lambda_{n} \nabla f_{\mid S}\left(\tau_{n}\right)+\mu_{n} \nabla \rho_{\mid S}\left(\tau_{n}\right),
$$

with $\mu_{n}<0$. Using the same arguments as above, we find that $\nabla g_{\mid V}(\tau)=$ $\mu \nabla \rho_{\mid S}(\tau)$ with $\mu \leq 0$ and $g(\tau) \geq 0$. This contradicts the remark after Lemma 3.4. Of course, this proof works for $\delta<0$.

Let $\Gamma_{g, \rho}$ be the following polar set:

$$
\Gamma_{g, \rho}=\{x \in U \mid \operatorname{rank}[\nabla g(x), \nabla \rho(x)]<2\} .
$$

By Lemma 2.5 and Lemma 2.1, we can assume that $\Gamma_{g, \rho} \backslash\{g=0\}$ does not intersect $X^{f} \backslash\{0\}$ in a neighborhood of 0 and so $\Gamma_{g, \rho} \backslash\{g=0\}$ does not intersect $X^{f} \cap \tilde{S}_{\epsilon}$ for $\epsilon>0$ sufficiently small. Since the critical points of $g_{\mid X^{f} \cap \tilde{S}_{\epsilon}}$ lie outside $\{g=0\}$, they do not belong to $\Gamma_{g, \rho} \cap \tilde{S}_{\epsilon}$ and so the critical points of $g_{\mid f^{-1}(\delta) \cap X \cap \tilde{S}_{\epsilon}}$ do not neither if $\delta$ is sufficiently small. Hence at each critical point of $g_{\mid f^{-1}(\delta) \cap X \cap \tilde{S_{\epsilon}}}, g_{\mid \tilde{S}_{\epsilon}}$ is a submersion. We are in position to apply Theorem 3.1 and Lemma 2.1 in [15]. For $0<|\delta| \ll \epsilon \ll 1$, we set

$$
\begin{gathered}
I(\delta, \epsilon, g)=\sum_{i=1}^{r} \operatorname{ind}\left(g, X \cap f^{-1}(\delta), p_{i}^{\delta, \epsilon}\right), \\
I(\delta, \epsilon,-g)=\sum_{i=1}^{r} \operatorname{ind}\left(-g, X \cap f^{-1}(\delta), p_{i}^{\delta, \epsilon}\right) .
\end{gathered}
$$


Theorem 3.8. We have

$$
\begin{aligned}
I(\delta, \epsilon, g)+I(\delta, \epsilon,-g)= & 2 \chi\left(X \cap f^{-1}(\delta) \cap \tilde{B}_{\epsilon}\right) \\
& -\chi\left(X \cap f^{-1}(\delta) \cap \tilde{S}_{\epsilon}\right)-\chi\left(X^{g} \cap f^{-1}(\delta) \cap \tilde{S}_{\epsilon}\right) .
\end{aligned}
$$

Proof. Let us denote by $\left\{a_{j}^{+}\right\}_{j=1}^{\alpha^{+}}$(resp. $\left\{a_{j}^{-}\right\}_{j=1}^{\alpha^{-}}$) the outwards-pointing (resp. inwards-pointing) critical points of $g: X \cap f^{-1}(\delta) \cap \tilde{S}_{\epsilon} \rightarrow \mathbb{R}$. Applying Morse theory type theorem ([15], Theorem 3.1) and using Lemma 2.1 in [15], we can write

$$
\begin{gathered}
I(\delta, \epsilon, g)+\sum_{j=1}^{\alpha^{-}} \operatorname{ind}\left(g, X \cap f^{-1}(\delta) \cap \tilde{S}_{\epsilon}, a_{j}^{-}\right)=\chi\left(X \cap f^{-1}(\delta) \cap \tilde{B}_{\epsilon}\right) \\
I(\delta, \epsilon,-g)+\sum_{j=1}^{\alpha^{+}} \operatorname{ind}\left(-g, X \cap f^{-1}(\delta) \cap \tilde{S}_{\epsilon},-a_{j}^{+}\right)=\chi\left(X \cap f^{-1}(\delta) \cap \tilde{B}_{\epsilon}\right)
\end{gathered}
$$

Let us evaluate

$$
\sum_{j=1}^{\alpha^{-}} \operatorname{ind}\left(g, X \cap f^{-1}(\delta) \cap \tilde{S}_{\epsilon}, a_{j}^{-}\right)+\sum_{j=1}^{\alpha^{+}} \operatorname{ind}\left(-g, X \cap f^{-1}(\delta) \cap \tilde{S}_{\epsilon}, a_{j}^{+}\right) .
$$

Since the outwards-pointing critical points of $g_{\mid X \cap f^{-1}(\delta) \cap \tilde{S}_{\epsilon}}$ lie in $\{g>0\}$ and the inwards-pointing critical points of $g_{\mid X \cap f^{-1}(\delta) \cap \tilde{S}_{\epsilon}}$ lie in $\{g<0\}$, we have

$$
\begin{array}{r}
\chi\left(X \cap f^{-1}(\delta) \cap \tilde{S}_{\epsilon} \cap\{g \geq 0\}\right)-\chi\left(X \cap f^{-1}(\delta) \cap \tilde{S}_{\epsilon} \cap\{g=0\}\right)= \\
\sum_{j=1}^{\alpha^{+}} \operatorname{ind}\left(g, X \cap f^{-1}(\delta) \cap \tilde{S}_{\epsilon}, a_{j}^{+}\right)
\end{array}
$$

and

$$
\begin{array}{r}
\chi\left(X \cap f^{-1}(\delta) \cap \tilde{S}_{\epsilon} \cap\{g \leq 0\}\right)-\chi\left(X \cap f^{-1}(\delta) \cap \tilde{S}_{\epsilon} \cap\{g=0\}\right)= \\
\sum_{j=1}^{\alpha^{-}} \operatorname{ind}\left(-g, X \cap f^{-1}(\delta) \cap \tilde{S}_{\epsilon}, a_{j}^{-}\right)
\end{array}
$$

Therefore making $(3)+(4)$ and using the Mayer-Vietoris sequence, we find

$$
\begin{aligned}
& \chi\left(X \cap f^{-1}(\delta) \cap \tilde{S}_{\epsilon}\right)-\chi\left(X \cap f^{-1}(\delta) \cap \tilde{S}_{\epsilon} \cap\{g=0\}\right)= \\
& \sum_{j=1}^{\alpha^{+}} \operatorname{ind}\left(g, X \cap f^{-1}(\delta) \cap \tilde{S}_{\epsilon}, a_{j}^{+}\right)+\sum_{j=1}^{\alpha^{-}} \operatorname{ind}\left(-g, X \cap f^{-1}(\delta) \cap \tilde{S}_{\epsilon}, a_{j}^{-}\right)
\end{aligned}
$$

Moreover we have

$$
\chi\left(X \cap f^{-1}(\delta) \cap \tilde{S}_{\epsilon}\right)=\sum_{j=1}^{\alpha^{+}} \operatorname{ind}\left(g, X \cap f^{-1}(\delta) \cap \tilde{S}_{\epsilon}, a_{j}^{+}\right)
$$




$$
\begin{gathered}
+\sum_{j=1}^{\alpha^{-}} \operatorname{ind}\left(g, X \cap f^{-1}(\delta) \cap \tilde{S}_{\epsilon}, a_{j}^{-}\right) \\
\chi\left(X \cap f^{-1}(\delta) \cap \tilde{S}_{\epsilon}\right)=\sum_{j=1}^{\alpha^{+}} \operatorname{ind}\left(-g, X \cap f^{-1}(\delta) \cap \tilde{S}_{\epsilon}, a_{j}^{+}\right) \\
+\sum_{j=1}^{\alpha^{-}} \operatorname{ind}\left(-g, X \cap f^{-1}(\delta) \cap \tilde{S}_{\epsilon}, a_{j}^{-}\right)
\end{gathered}
$$

The combination $-(5)+(6)+(7)$ leads to

$$
\begin{aligned}
& \chi\left(X \cap f^{-1}(\delta) \cap \tilde{S}_{\epsilon}\right)+\chi\left(X \cap f^{-1}(\delta) \cap \tilde{S}_{\epsilon} \cap\{g=0\}\right)= \\
& \quad \sum_{j=1}^{\alpha^{+}} \operatorname{ind}\left(-g, X \cap f^{-1}(\delta) \cap \tilde{S}_{\epsilon}, a_{j}^{+}\right)+\sum_{j=1}^{\alpha^{-}} \operatorname{ind}\left(g, X \cap f^{-1}(\delta) \cap \tilde{S}_{\epsilon}, a_{j}^{-}\right) .
\end{aligned}
$$

Let us assume now that $(X, 0)$ is equipped with a Whitney stratification $\mathcal{W}=\cup_{\alpha \in A} W_{\alpha}$ and $f:(X, 0) \rightarrow(\mathbb{R}, 0)$ has an isolated critical point at 0 . In this situation, our results apply taking for $\mathcal{V}$ the following stratification:

$$
\left\{W_{\alpha} \backslash f^{-1}(0), W_{\alpha} \cap f^{-1}(0) \backslash\{0\},\{0\} \mid W_{\alpha} \in \mathcal{W}\right\} .
$$

Corollary 3.9. If $f:(X, 0) \rightarrow(\mathbb{R}, 0)$ has an isolated stratified critical point at 0 , then

$$
\begin{aligned}
& I(\delta, \epsilon, g)+I(\delta, \epsilon,-g)=2 \chi\left(X \cap f^{-1}(\delta)\right.\left.\cap \tilde{B}_{\epsilon}\right) \\
&-\chi\left(X^{f} \cap \tilde{S}_{\epsilon}\right)-\chi\left(X^{f} \cap X^{g} \cap \tilde{S}_{\epsilon}\right) .
\end{aligned}
$$

Proof. For each stratum $W$ of $X$, let

$$
\Gamma_{f, \rho}^{W}=\left\{x \in W \mid \operatorname{rank}\left[\nabla f_{\mid W}(x), \nabla \rho_{\mid W}(x)\right]<2\right\},
$$

and let $\Gamma_{f, \rho}=\cup_{W} \Gamma_{f, \rho}^{W}$. By Lemma 3.4 applied to $X$ and $f$ instead of $X^{f}$ and $g, \Gamma_{f, \rho} \cap\{f=0\} \subset\{0\}$ in a neighborhood of the origin and so 0 is a regular value of $f: X \cap \tilde{S}_{\epsilon} \rightarrow \mathbb{R}$ for $\epsilon$ sufficiently small. By Thom-Mather's second isotopy lemma, $X \cap f^{-1}(0) \cap \tilde{S}_{\epsilon}$ is homeomorphic to $X \cap f^{-1}(\delta) \cap \tilde{S}_{\epsilon}$ for $\delta$ sufficiently small.

Now let $p$ be a stratified critical point of $f: X^{g} \rightarrow \mathbb{R}$. By Lemma 2.1, we know that $p$ belongs to $f^{-1}(0) \cap X^{g}$ and so $p$ is also a critical point of $g: X^{f} \rightarrow \mathbb{R}$. Hence $p=0$ by Condition (A), and $f: X^{g} \rightarrow \mathbb{R}$ has an isolated stratified critical point at 0 . As above, we conclude that $X^{f} \cap X^{g} \cap \tilde{S}_{\epsilon}$ is homeomorphic to $X^{g} \cap f^{-1}(\delta) \cap \tilde{S}_{\epsilon}$.

Let $\omega(x)=\sqrt{x_{1}^{2}+\cdots+x_{n}^{2}}$ be the euclidian distance to the origin. As explained by Durfee in [10], Lemma 1.8 and Lemma 3.6, there is a neighborhood $\Omega$ of 0 in $\mathbb{R}^{n}$ such that for every stratum $V$ of $X^{f}, \nabla \omega_{\mid V}$ and $\nabla \rho_{\mid V}$ are non-zero and do not point in opposite direction in $\Omega \backslash\{0\}$. Applying 
Durfee's argument (10, Proposition 1.7 and Proposition 3.5), we see that $X^{f} \cap \tilde{S}_{\epsilon}$ is homeomorphic to $X^{f} \cap S_{\epsilon^{\prime}}$ for $\epsilon, \epsilon^{\prime}>0$ sufficiently small. Similarly $X^{f} \cap X^{g} \cap \tilde{S}_{\epsilon}$ and $X^{f} \cap X^{g} \cap S_{\epsilon^{\prime}}$ are homemorphic. Now let us compare $X \cap f^{-1}(\delta) \cap \tilde{B}_{\epsilon}$ and $X \cap f^{-1}(\delta) \cap B_{\epsilon^{\prime}}$. Let us choose $\epsilon^{\prime}$ and $\epsilon$ such that

$$
X \cap f^{-1}(\delta) \cap B_{\epsilon^{\prime}} \subset X \cap f^{-1}(\delta) \cap \tilde{B}_{\epsilon} \subset \Omega .
$$

If $\delta$ is sufficiently small then, for every stratum $V \nsubseteq X^{f}, \nabla \omega_{\mid V \cap f^{-1}(\delta)}$ and $\nabla \rho_{\mid V \cap f^{-1}(\delta)}$ are non-zero and do not point in opposite direction in $\tilde{B}_{\epsilon} \backslash B_{\epsilon^{\prime}}$. Otherwise, by Thom's $\left(a_{f}\right)$-condition, we would find a point $p$ in $X^{f} \cap\left(\tilde{B}_{\epsilon}\right)$ $\left.B_{\epsilon^{\prime}}\right)$ such that either $\nabla \omega_{\mid S}(p)$ or $\nabla \rho_{\mid S}(p)$ vanish or $\nabla \omega_{\mid S}(p)$ and $\nabla \rho_{\mid S}(p)$ point in opposite direction, where $S$ is the stratum of $X^{f}$ that contains $p$. This is impossible if we are sufficiently close to the origin. Now, applying the same arguments as Durfee [10, Proposition 1.7 and Proposition 3.5, we see that $X \cap f^{-1}(\delta) \cap \tilde{B}_{\epsilon}$ is homeomorphic to $X \cap f^{-1}(\delta) \cap B_{\epsilon^{\prime}}$ and that $X \cap f^{-1}(\delta) \cap \tilde{S}_{\epsilon}$ is homeomorphic to $X \cap f^{-1}(\delta) \cap S_{\epsilon^{\prime}}$.

Theorem 3.10. We have

$I(\delta, \epsilon, g)+I(\delta, \epsilon,-g)=2 \chi\left(M_{f}^{\delta, \epsilon}\right)-\chi\left(X \cap f^{-1}(\delta) \cap S_{\epsilon}\right)-\chi\left(X^{g} \cap f^{-1}(\delta) \cap S_{\epsilon}\right)$.

Corollary 3.11. If $f:(X, 0) \rightarrow(\mathbb{R}, 0)$ has an isolated stratified critical point at 0 , then

$$
I(\delta, \epsilon, g)+I(\delta, \epsilon,-g)=2 \chi\left(M_{f}^{\delta, \epsilon}\right)-\chi\left(\operatorname{Lk}\left(X^{f}\right)\right)-\chi\left(\operatorname{Lk}\left(X^{f} \cap X^{g}\right)\right) .
$$

Let us remark if $\operatorname{dim} X=2$ then in Theorem 3.10 and in Corollary 3.11, the last term of the right-hand side of the equality vanishes. If $\operatorname{dim} X=1$ then in Theorem 3.10 and in Corollary 3.11, the last two terms of the righthand side of the equality vanish.

\section{An infinitesimal Gauss-Bonnet formula}

In this section, we apply the results of the previous section to the case of linear forms and we establish a Gauss-Bonnet type formula for the real Milnor fibre.

We will first show that generic linear forms satisfy Condition (A) and Condition (B). For $v \in S^{n-1}$, let us denote by $v^{*}$ the function $v^{*}(x)=\langle v, x\rangle$.

Lemma 4.1. There exists a subanalytic set $\Sigma_{1} \subset S^{n-1}$ of positive codimension such that if $v \notin \Sigma_{1},\left\{v^{*}=0\right\}$ intersects $X \backslash\{0\}$ transversally (in the stratified sense) in a neighborhood of the origin.

Proof. It is a particular case of Lemma 3.8 in [14].

Corollary 4.2. If $v \notin \Sigma_{1}$ then $v_{\mid X}^{*}:(X, 0) \rightarrow(\mathbb{R}, 0)$ has an isolated stratified point at 0 . 
Proof. By Lemma 2.1, we know that the stratified critical points of $v_{\mid X}^{*}$ lie in $\left\{v^{*}=0\right\}$. But since $\left\{v^{*}=0\right\}$ intersects $X \backslash\{0\}$ transversally, the only possible critical point of $v_{\mid X}^{*}:(X, 0) \rightarrow(\mathbb{R}, 0)$ is the origin.

Lemma 4.3. There exists a subanalytic set $\Sigma_{2} \subset S^{n-1}$ of positive codimension such that if $v \notin \Sigma_{2}$, then $\Gamma_{f, v^{*}}$ is a $C^{1}$-subanalytic curve (possibly empty) in a neighborhood of 0.

Proof. Let $V$ be stratum of dimension $e$ such that $V \nsubseteq X^{f}$. We can assume that $e \geq 2$. Let

$$
M_{V}=\left\{(x, y) \in V \times \mathbb{R}^{n} \mid \operatorname{rank}\left[\nabla f_{\mid V}(x), \nabla y_{\mid V}^{*}(x)\right]<2\right\} .
$$

It is a subanalytic manifold of class $C^{1}$ and of dimension $n+1$. To see this, let us pick a point $(x, y)$ in $M_{V}$. In a neighborhood of $x, V$ is defined by the vanishing of $k=n-e C^{2}$-subanalytic functions $f_{1}, \ldots, f_{k}$. Since $V$ is not included in $X^{f}, f: V \rightarrow \mathbb{R}$ is a submersion and we can assume that in a neighborhood of $x$, the following $(k+1) \times(k+1)$-minor:

$$
\frac{\partial\left(f_{1}, \ldots, f_{k}, f\right)}{\partial\left(x_{1}, \ldots, x_{k}, x_{k+1}\right)}
$$

does not vanish. Therefore, in a neighborhood of $(x, y), M_{V}$ is defined by the vanishing of the following $(k+2) \times(k+2)$-minors:

$$
\frac{\partial\left(f_{1}, \ldots, f_{k}, f, y^{*}\right)}{\partial\left(x_{1}, \ldots, x_{k}, x_{k+1}, x_{k+2}\right)}, \ldots, \frac{\partial\left(f_{1}, \ldots, f_{k}, f, y^{*}\right)}{\partial\left(x_{1}, \ldots, x_{k}, x_{k+1}, x_{n}\right)} .
$$

A simple computation of determinants shows that the gradient vectors of these minors are linearly independent. As in previous lemmas, we show that $\Sigma_{f, v^{*}}$ is one-dimensional considering the projection

$$
\begin{aligned}
\pi_{2}: M^{V} & \rightarrow \mathbb{R}^{n} \\
& (x, y)
\end{aligned}
$$

Since $\Gamma_{f, v^{*}}=\cup_{V \nsubseteq X_{f}} \Gamma_{f, v^{*}}^{V}$, we get the result.

Let $\Sigma=\Sigma_{1} \cup \Sigma_{2}$, it is a subanalytic subset of $S^{n-1}$ of positive codimension and if $v \notin \Sigma$ then $v^{*}$ satisfies Conditions (A) and (B). In particular, $v_{\mid f^{-1}(\delta) \cap X \cap B_{\epsilon}}^{*}$ has a finite number of critical points $p_{1}^{\delta, \epsilon}, \ldots, p_{r_{v}}^{\delta, \epsilon}$. We recall that

$$
\begin{gathered}
I\left(\delta, \epsilon, v^{*}\right)=\sum_{i=1}^{r_{v}} \operatorname{ind}\left(v^{*}, X \cap f^{-1}(\delta), p_{i}^{\delta, \epsilon}\right), \\
I\left(\delta, \epsilon,-v^{*}\right)=\sum_{i=1}^{r_{v}} \operatorname{ind}\left(-v^{*}, X \cap f^{-1}(\delta), p_{i}^{\delta, \epsilon}\right) .
\end{gathered}
$$

In this situation, Theorem 3.10 and Corollary 3.11 become 
Corollary 4.4. If $v \notin \Sigma$ then

$I\left(\delta, \epsilon, v^{*}\right)+I\left(\delta, \epsilon,-v^{*}\right)=2 \chi\left(M_{f}^{\delta, \epsilon}\right)-\chi\left(X \cap f^{-1}(\delta) \cap S_{\epsilon}\right)-\chi\left(X^{v^{*}} \cap f^{-1}(\delta) \cap S_{\epsilon}\right)$.

Furthermore, if $f:(X, 0) \rightarrow(\mathbb{R}, 0)$ has an isolated stratified critical point at 0 , then

$$
I\left(\delta, \epsilon, v^{*}\right)+I\left(\delta, \epsilon,-v^{*}\right)=2 \chi\left(M_{f}^{\delta, \epsilon}\right)-\chi\left(\operatorname{Lk}\left(X^{f}\right)\right)-\chi\left(\operatorname{Lk}\left(X^{f} \cap X^{v^{*}}\right)\right) .
$$

As an application, we give a Gauss-Bonnet formula for the Milnor fibre $M_{f}^{\delta, \epsilon}$. Let $\Lambda_{0}\left(X \cap f^{-1}(\delta),-\right)$ be the Gauss-Bonnet measure on $X \cap f^{-1}(\delta)$ defined by

$$
\Lambda_{0}\left(X \cap f^{-1}(\delta), U^{\prime}\right)=\frac{1}{s_{n-1}} \int_{S^{n-1}} \sum_{x \in U^{\prime}} \operatorname{ind}\left(v^{*}, X \cap f^{-1}(\delta), x\right) d x,
$$

where $U^{\prime}$ is a Borel set of $X \cap f^{-1}(\delta)$ (see [6], page 299) and $s_{n-1}$ is the volume of the unit sphere $S^{n-1}$. Note that if $x$ is not a critical point of $v_{\mid X \cap f^{-1}(\delta)}^{*}$ then $\operatorname{ind}\left(v^{*}, X \cap f^{-1}(\delta), x\right)=0$. We are going to evaluate

$$
\lim _{\epsilon \rightarrow 0} \lim _{\delta \rightarrow 0} \Lambda_{0}\left(X \cap f^{-1}(\delta), M_{f}^{\delta, \epsilon}\right) .
$$

Theorem 4.5. We have

$$
\begin{aligned}
\lim _{\epsilon \rightarrow 0} \lim _{\delta \rightarrow 0} \Lambda_{0}\left(X \cap f^{-1}(\delta),\right. & \left.M_{f}^{\delta, \epsilon}\right)=\chi\left(M_{f}^{\delta, \epsilon}\right)-\frac{1}{2} \chi\left(X \cap f^{-1}(\delta) \cap S_{\epsilon}\right) \\
& -\frac{1}{2 s_{n-1}} \int_{S^{n-1}} \chi\left(X \cap f^{-1}(\delta) \cap\left\{v^{*}=0\right\} \cap S_{\epsilon}\right) d v .
\end{aligned}
$$

Furthermore, if $f:(X, 0) \rightarrow(\mathbb{R}, 0)$ has an isolated stratified critical point at 0 , then

$$
\begin{aligned}
\lim _{\epsilon \rightarrow 0} \lim _{\delta \rightarrow 0} \Lambda_{0}\left(X \cap f^{-1}(\delta), M_{f}^{\delta, \epsilon}\right)=\chi\left(M_{f}^{\delta, \epsilon}\right)-\frac{1}{2} \chi\left(\operatorname{Lk}\left(X^{f}\right)\right) & \\
& -\frac{1}{2 s_{n-1}} \int_{S^{n-1}} \chi\left(\operatorname{Lk}\left(X^{f} \cap X^{v^{*}}\right)\right) d v .
\end{aligned}
$$

Proof. By definition, we have

$$
\Lambda_{0}\left(X \cap f^{-1}(\delta), M_{f}^{\delta, \epsilon}\right)=\frac{1}{s_{n-1}} \int_{S^{n-1}} \sum_{x \in M_{f}^{\delta, \epsilon}} \operatorname{ind}\left(v^{*}, X \cap f^{-1}(\delta), x\right) d v .
$$

It is not difficult to see that

$$
\begin{aligned}
& \Lambda_{0}\left(X \cap f^{-1}(\delta), M_{f}^{\delta, \epsilon}\right)= \\
& \frac{1}{2 s_{n-1}} \int_{S^{n-1}}\left[\sum_{x \in M_{f}^{\delta, \epsilon}} \operatorname{ind}\left(v^{*}, X \cap f^{-1}(\delta), x\right)+\operatorname{ind}\left(-v^{*}, X \cap f^{-1}(\delta), x\right)\right] d v .
\end{aligned}
$$


Note that if $v \notin \Sigma$ then

$$
\sum_{x \in M_{f}^{\delta, \epsilon}} \operatorname{ind}\left(v^{*}, X \cap f^{-1}(\delta), x\right)+\operatorname{ind}\left(-v^{*}, X \cap f^{-1}(\delta), x\right)
$$

is equal to $I\left(\delta, \epsilon, v^{*}\right)+I\left(\delta, \epsilon,-v^{*}\right)$ and is uniformly bounded by Hardt's theorem. By Lebesgue's theorem, we obtain

$$
\begin{aligned}
& \lim _{\epsilon \rightarrow 0} \lim _{\delta \rightarrow 0} \Lambda_{0}\left(X \cap f^{-1}(\delta), M_{f}^{\delta, \epsilon}\right)= \\
& \frac{1}{2 s_{n-1}} \int_{S^{n-1}} \lim _{\epsilon \rightarrow 0} \lim _{\delta \rightarrow 0}\left[I\left(\delta, \epsilon, v^{*}\right)+I\left(\delta, \epsilon,-v^{*}\right)\right] d v .
\end{aligned}
$$

We just have to apply the previous corollary to conclude.

\section{INFINITESIMAL LINEAR KINEMATIC FORMULAS}

In this section, we apply the results of the previous section to the case of a linear function in order to obtain "infinitesimal" linear kinematic formulas for closed subanalytic germs.

We start recalling known facts on the geometry of subanalytic sets. We need some notations:

- for $k \in\{0, \ldots, n\}, G_{n}^{k}$ is the Grassmann manifold of $k$-dimensional linear subspaces in $\mathbb{R}^{n}$ and $g_{n}^{k}$ is its volume,

- for $k \in \mathbb{N}, b_{k}$ is the volume of the $k$-dimensional unit ball and $s_{k}$ is the volume of the $k$-dimensional unit sphere.

In [17], Fu developed integral geometry for compact subanalytic sets. Using the technology of the normal cycle, he associated with every compact subanalytic set $X \subset \mathbb{R}^{n}$ a sequence of curvature measures

$$
\Lambda_{0}(X,-), \ldots, \Lambda_{n}(X,-),
$$

called the Lipschitz-Killing measures. He proved several integral geometry formulas, among them a Gauss-Bonnet formula and a kinematic formula. Later another description of the measures using stratified Morse theory was given by Broecker and Kuppe [6] (see also [5]). The reader can refer to [14], Section 2, for a rather complete presentation of these two approaches and for the definition of the Lipschitz-Killing measures.

Let us give some comments on these Lipschitz-Killing curvatures. If dim $X=d$ then

$$
\Lambda_{d+1}\left(X, U^{\prime}\right)=\cdots=\Lambda_{n}\left(X, U^{\prime}\right)=0,
$$

for any Borel set $U^{\prime}$ of $X$ and $\Lambda_{d}\left(X, U^{\prime}\right)=\mathcal{L}_{d}\left(U^{\prime}\right)$, where $\mathcal{L}_{d}$ is the $d$ dimensional Lebesgue measure in $\mathbb{R}^{n}$. Furthemore if $X$ is smooth then for any Borel set $U^{\prime}$ of $X$ and for $k \in\{0, \ldots, d\}, \Lambda_{k}\left(X, U^{\prime}\right)$ is related to the classical Lipschitz-Killing-Weil curvature $K_{d-k}$ through the following equality:

$$
\Lambda_{k}\left(X, U^{\prime}\right)=\frac{1}{s_{n-d-k-1}} \int_{U^{\prime}} K_{d-k}(x) d x
$$


In 14, Section 5, we studied the asymptotic behaviour of the LipschitzKilling measures in the neighborhood of a point of $X$. Namely we proved the following theorem ([14, Theorem 5.1).

Theorem 5.1. Let $X \subset \mathbb{R}^{n}$ be a closed subanalytic set such that $0 \in X$. We have:

$$
\lim _{\epsilon \rightarrow 0} \Lambda_{0}\left(X, X \cap B_{\epsilon}\right)=1-\frac{1}{2} \chi(\operatorname{Lk}(X))-\frac{1}{2 g_{n}^{n-1}} \int_{G_{n}^{n-1}} \chi(\operatorname{Lk}(X \cap H)) d H .
$$

Furthermore for $k \in\{1, \ldots, n-2\}$, we have:

$$
\begin{aligned}
\lim _{\epsilon \rightarrow 0} \frac{\Lambda_{k}\left(X, X \cap B_{\epsilon}\right)}{b_{k} \epsilon^{k}}=-\frac{1}{2 g_{n}^{n-k-1}} \int_{G_{n}^{n-k-1}} \chi(\operatorname{Lk}(X \cap H)) d H \\
+\frac{1}{2 g_{n}^{n-k+1}} \int_{G_{n}^{n-k+1}} \chi(\operatorname{Lk}(X \cap L)) d L,
\end{aligned}
$$

and:

$$
\begin{gathered}
\lim _{\epsilon \rightarrow 0} \frac{\Lambda_{n-1}\left(X, X \cap B_{\epsilon}\right)}{b_{n-1} \epsilon^{n-1}}=\frac{1}{2 g_{n}^{2}} \int_{G_{n}^{2}} \chi(\operatorname{Lk}(X \cap H)) d H, \\
\lim _{\epsilon \rightarrow 0} \frac{\Lambda_{n}\left(X, X \cap B_{\epsilon}\right)}{b_{n} \epsilon^{n}}=\frac{1}{2 g_{n}^{1}} \int_{G_{n}^{1}} \chi(\operatorname{Lk}(X \cap H)) d H .
\end{gathered}
$$

In the sequel, we will use these equalities and Theorem 4.5 to establish linear kinematic types formulas for the quantities $\lim _{\epsilon \rightarrow 0} \frac{\Lambda_{k}\left(X, X \cap B_{\epsilon}\right)}{b_{k} \epsilon^{k}}, k=$ $1, \ldots, n$. Let us start with some lemmas. We work with a closed subanalytic set $X$ such that $0 \in X$, equipped with a Whitney stratification $\left\{W_{\alpha}\right\}_{\alpha \in A}$.

Lemma 5.2. Let $f$ be a $C^{2}$-subanalytic function such that $f_{\mid X}: X \rightarrow \mathbb{R}$ has an isolated stratified critical point at 0 . Then for $0<\delta \ll \epsilon \ll 1$, we have

$$
\chi\left(M_{f}^{\delta, \epsilon}\right)+\chi\left(M_{f}^{-\delta, \epsilon}\right)=\chi(\operatorname{Lk}(X))+\chi\left(\operatorname{Lk}\left(X^{f}\right)\right) .
$$

Proof. With the same technics and arguments as the ones we used in order to establish Corollary 3.11, we can prove that

$$
\operatorname{ind}(f, X, 0)+\operatorname{ind}(-f, X, 0)=2 \chi\left(X \cap B_{\epsilon}\right)-\chi(\operatorname{Lk}(X))-\chi\left(\operatorname{Lk}\left(X^{f}\right)\right) .
$$

We conclude thanks to the following equalities

$$
\operatorname{ind}(f, X, 0)=1-\chi\left(M_{f}^{-\delta, \epsilon}\right), \operatorname{ind}(-f, X, 0)=1-\chi\left(M_{f}^{\delta, \epsilon}\right),
$$

and

$$
\chi\left(X \cap B_{\epsilon}\right)=1 .
$$

Corollary 5.3. There exist a subanalytic set $\Sigma_{1} \subset S^{n-1}$ of positive codimension such that if $v \notin \Sigma$ then for $0<\delta \ll \epsilon \ll 1$,

$$
\chi\left(M_{v^{*}}^{\delta, \epsilon}\right)+\chi\left(M_{v^{*}}^{-\delta, \epsilon}\right)=\chi(\operatorname{Lk}(X))+\chi\left(\operatorname{Lk}\left(X \cap\left\{v^{*}=0\right\}\right)\right) .
$$

Proof. Apply Corollary 4.2 and Lemma 5.2 . 
Lemma 5.4. Let $S \subset \mathbb{R}^{n}$ be $C^{2}$-subanalytic manifold. Let $H \in G_{n}^{n-k}$, $k \in\{1, \ldots, n\}$ and let $G_{H^{\perp}}^{1}$ be the Grassmann manifold of lines in the orthogonal complement $H^{\perp}$ of $H$. There exists a subanalytic set $\Sigma_{H}^{\prime} \subset G_{H^{\perp}}^{1}$ of positive codimension such that if $\nu \notin \Sigma_{H}^{\prime}$ then $H \oplus \nu$ intersects $S \backslash\{0\}$ transversally.

Proof. Assume that $S$ has dimension $e$ and that $H$ is given by the equations $x_{1}=\ldots=x_{k}=0$ so that $H^{\perp}=\mathbb{R}^{k}$ with coordinate system $\left(x_{1}, \ldots, x_{k}\right)$. Let $W$ be defined by

$$
\begin{aligned}
& W=\left\{\left(x, v_{1}, \ldots, v_{k-1}\right) \in \mathbb{R}^{n} \times\left(\mathbb{R}^{k}\right)^{k-1} \mid x \in S \backslash\{0\}\right. \\
& \text { and } \left.\left\langle x, v_{1}\right\rangle=\cdots=\left\langle x, v_{k-1}\right\rangle=0\right\},
\end{aligned}
$$

where $v_{i} \in \mathbb{R}^{k} \times\{0\} \subset \mathbb{R}^{n}$. Let us show that $W$ is a $C^{2}$-subanalytic manifold of dimension $e+(k-1)^{2}$. Let $(y, w)$ be a point in $W$. We can assume that around $y, S$ is defined by the vanishing of $n-e C^{2}$-subanalytic functions $f_{1}, \ldots, f_{n-e}$. Hence in a neighborhood of $(y, w), W$ is defined by the equations:

$$
f_{1}(x)=\ldots=f_{n-e}(x)=0 \text { and }\left\langle x, v_{1}\right\rangle=\cdots=\left\langle x, v_{k-1}\right\rangle=0 .
$$

Because $y \neq 0$, we see that the gradient vectors of this $n-e+k-1$ functions are linearly independent at $(y, w)$. This enables us to conclude that $W$ is a $C^{2}$-subanalytic manifold of dimension $e+(k-1)^{2}$. Let $\pi_{2}$ be the following projection:

$$
\pi_{2}: W \rightarrow\left(\mathbb{R}^{n}\right)^{n-k},\left(x, v_{1}, \ldots, v_{n-k}\right) \mapsto\left(v_{1}, \ldots, v_{n-k}\right) .
$$

Bertini-Sard's theorem implies that the set of critical values of $\pi_{2}$ is a subanalytic set of positive codimension. If $\left(v_{1}, \ldots, v_{k-1}\right)$ lies outside this subanalytic set then the $(n-k+1)$-plane $\left\{x \in \mathbb{R}^{n} \mid\left\langle x, v_{1}\right\rangle=\cdots=\left\langle x, v_{k-1}\right\rangle=0\right\}$ contains $H$ and intersects $S \backslash\{0\}$ transversally.

Now we can present our infinitesimal linear kinematic formulas. Let $H \in$ $G_{n}^{n-k}, k \in\{1, \ldots, n\}$, and let $S_{H^{\perp}}^{k-1}$ be the unit sphere of the orthogonal complement of $H$. Let $v$ be an element in $S_{H^{\perp}}^{k-1}$. For $\delta>0$, we denote by $H_{v, \delta}$ the $(n-k)$-dimensional affine space $H+\delta v$ and we set

$$
\beta_{0}(H, v)=\lim _{\epsilon \rightarrow 0} \lim _{\delta \rightarrow 0} \Lambda_{0}\left(H_{\delta, v} \cap X, H_{\delta, v} \cap X \cap B_{\epsilon}\right) .
$$

Then we set

$$
\beta_{0}(H)=\frac{1}{s_{k-1}} \int_{S_{H^{\perp}}^{k-1}} \beta_{0}(H, v) d v .
$$

Theorem 5.5. For $k \in\{1, \ldots, n\}$, we have

$$
\lim _{\epsilon \rightarrow 0} \frac{\Lambda_{k}\left(X, X \cap B_{\epsilon}\right)}{b_{k} \epsilon^{k}}=\frac{1}{g_{n}^{n-k}} \int_{G_{n}^{n-k}} \beta_{0}(H) d H .
$$


Proof. We treat first the case $k \in\{1, \ldots, n-2\}$. By Theorem 5.1 , we know that

$$
\begin{aligned}
\lim _{\epsilon \rightarrow 0} \frac{\Lambda_{k}\left(X, X \cap B_{\epsilon}\right)}{b_{k} \epsilon^{k}}=-\frac{1}{2 g_{n}^{n-k-1}} \int_{G_{n}^{n-k-1}} \chi(\operatorname{Lk}(X \cap H)) d H \\
\quad+\frac{1}{2 g_{n}^{n-k+1}} \int_{G_{n}^{n-k+1}} \chi(\operatorname{Lk}(X \cap L)) d L .
\end{aligned}
$$

By Lemma 3.8 in [14], we know that generically $H$ intersects $X \backslash\{0\}$ transversally in a neighborhood of the origin. Let us fix $H$ that satisfies this generic property. For any $v \in S_{H^{\perp}}^{k-1}$, let $\nu$ be the line generated by $v$ and let $L_{v}$ be the $(n-k+1)$-plane defined by $L_{v}=H \oplus \nu$. By Lemma 5.4, we know that for $v$ generic in $S_{H^{\perp}}^{k-1}, L_{v}$ intersects $X \backslash\{0\}$ transversally in a neighborhood of the origin. Therefore, $v_{\mid X \cap L_{v}}^{*}$ has an isolated singular point at 0 and we can apply Theorem 4.5. We have

$$
\begin{aligned}
\lim _{\epsilon \rightarrow 0} \lim _{\delta \rightarrow 0} \Lambda_{0}\left(X \cap L_{v} \cap\left\{v^{*}=\delta\right\}, X \cap L_{v} \cap\left\{v^{*}=\delta\right\} \cap B_{\epsilon}\right)= \\
\chi\left(X \cap L_{v} \cap\left\{v^{*}=\delta\right\} \cap B_{\epsilon}\right)-\frac{1}{2} \chi\left(\operatorname{Lk}\left(X \cap L_{v} \cap\left\{v^{*}=0\right\}\right)\right) \\
-\frac{1}{2 s_{n-k}} \int_{S_{L_{v}}^{n-k}} \chi\left(\operatorname{Lk}\left(X \cap L_{v} \cap\left\{v^{*}=0\right\} \cap\left\{w^{*}=0\right\}\right)\right) d w,
\end{aligned}
$$

where $S_{L_{v}}^{n-k}$ is the unit sphere of $L_{v}$. Let us remark that $L_{v} \cap\left\{v^{*}=\delta\right\}$ is exactly $H_{v, \delta}$ and that $L_{v} \cap\left\{v^{*}=0\right\}$ is $H$. We can also apply Lemma 5.2 to $v_{\mid X \cap L_{v}}^{*}$ to obtain the following relation:

$$
\begin{aligned}
\beta_{0}(H, v)+\beta_{0}(H,-v)=\chi\left(\operatorname{Lk}\left(X \cap L_{v}\right)\right) & \\
& -\frac{1}{s_{n-k}} \int_{S_{L v}^{n-k}} \chi\left(\operatorname{Lk}\left(X \cap H \cap\left\{w^{*}=0\right\}\right)\right) d w .
\end{aligned}
$$

Since $\beta(H)$ is equal to

$$
\frac{1}{2 s_{k-1}} \int_{S_{H^{\perp}}^{k-1}}\left[\beta_{0}(H, v)+\beta_{0}(H,-v)\right] d v
$$

we find that

$$
\begin{aligned}
\beta(H)=\frac{1}{2 s_{k-1}} & \int_{S_{H^{\perp}}^{k-1}} \chi\left(\operatorname{Lk}\left(X \cap L_{v}\right)\right) d v \\
& -\frac{1}{2 s_{k-1} s_{n-k}} \int_{S_{H^{\perp}}^{k-1}} \int_{S_{L_{v}}^{n-k}} \chi\left(\operatorname{Lk}\left(X \cap H \cap\left\{w^{*}=0\right\}\right)\right) d w d v .
\end{aligned}
$$

Replacing spheres with Grassman manifolds in this equality, we obtain

$$
\begin{aligned}
\beta(H)=\frac{1}{2 g_{k}^{1}} & \int_{G_{H^{\perp}}^{1}} \chi(\operatorname{Lk}(X \cap H \oplus \nu)) d \nu \\
& -\frac{1}{2 g_{k}^{1} g_{n-k+1}^{n-k}} \int_{G_{H^{\perp}}^{1}} \int_{G_{H \oplus \nu}^{n-k}} \chi(\operatorname{Lk}(X \cap H \cap K)) d K d \nu .
\end{aligned}
$$


Therefore, we have

$$
\begin{array}{r}
\frac{1}{g_{n}^{n-k}} \int_{G_{n}^{n-k}} \beta(H) d H=\frac{1}{2 g_{k}^{1} g_{n}^{n-k}} \int_{G_{n}^{n-k}} \int_{G_{H^{\perp}}^{1}} \chi(\operatorname{Lk}(X \cap H \oplus \nu)) d \nu d H- \\
\frac{1}{2 g_{n}^{n-k} g_{k}^{1} g_{n-k+1}^{n-k}} \int_{G_{n}^{n-k}} \int_{G_{H^{\perp}}^{1}} \int_{G_{H \oplus \nu}^{n-k}} \chi(\operatorname{Lk}(X \cap H \cap K)) d K d \nu d H .
\end{array}
$$

Let us compute

$$
\mathcal{I}=\frac{1}{2 g_{n}^{n-k} g_{k}^{1}} \int_{G_{n}^{n-k}} \int_{G_{H^{\perp}}^{1}} \chi(\operatorname{Lk}(X \cap H \oplus \nu)) d \nu d H .
$$

Let $\mathcal{H}$ be the flag variety of pairs $(L, H), L \in G_{n}^{n-k+1}$ and $H \in G_{L}^{n-k}$. This variety is a bundle over $G_{n}^{n-k}$, each fibre being a $G_{k}^{1}$. Hence we have

$$
\begin{aligned}
\int_{G_{n}^{n-k}} \int_{G_{H^{\perp}}^{1}} \chi(\operatorname{Lk}(X \cap H \oplus \nu)) d \nu d H= & \int_{G_{n}^{n-k+1}} \int_{G_{L}^{n-k}} \chi(\operatorname{Lk}(X \cap L)) d H d L= \\
& g_{n-k+1}^{n-k} \int_{G_{n}^{n-k+1}} \chi(\operatorname{Lk}(X \cap L)) d L .
\end{aligned}
$$

Finally, we get that

$$
\begin{aligned}
& \mathcal{I}=\frac{g_{n-k+1}^{n-k}}{2 g_{n}^{n-k} g_{k}^{1}} \int_{G_{n}^{n-k+1}} \chi(\operatorname{Lk}(X \cap L)) d L= \\
& \frac{1}{2 g_{n}^{n-k+1}} \int_{G_{n}^{n-k+1}} \chi(\operatorname{Lk}(X \cap L)) d L .
\end{aligned}
$$

Let us compute now

$$
\mathcal{J}=\frac{1}{2 g_{n}^{n-k} g_{k}^{1} g_{n-k+1}^{n-k}} \int_{G_{n}^{n-k}} \int_{G_{H}^{1} \perp} \int_{G_{H \oplus \nu}^{n-k}} \chi(\operatorname{Lk}(X \cap H \cap K)) d K d \nu d H .
$$

First, as we have just done above, we can write

$$
\mathcal{J}=\frac{1}{2 g_{n}^{n-k} g_{k}^{1} g_{n-k+1}^{n-k}} \int_{G_{n}^{n-k+1}} \int_{G_{L}^{n-k}} \int_{G_{L}^{n-k}} \chi(\operatorname{Lk}(X \cap H \cap K)) d K d H d L .
$$

Then we remark (see [14], Corollary 3.11 for a similar argument) that

$$
\frac{1}{g_{n-k+1}^{n-k}} \int_{G_{L}^{n-k}} \chi(\operatorname{Lk}(X \cap H \cap K)) d K=\frac{1}{g_{n-k}^{n-k-1}} \int_{G_{H}^{n-k-1}} \chi(\operatorname{Lk}(X \cap J)) d J,
$$

and so

$$
\mathcal{J}=\frac{1}{2 g_{n}^{n-k} g_{k}^{1} g_{n-k}^{n-k-1}} \int_{G_{n}^{n-k+1}} \int_{G_{L}^{n-k}} \int_{G_{H}^{n-k-1}} \chi(\operatorname{Lk}(X \cap J)) d J d H d L .
$$

Considering the flag variety of pairs $(H, J), H \in G_{L}^{n-k}$ and $J \in G_{H}^{n-k-1}$, and proceeding as above, we find

$$
\int_{G_{L}^{n-k}} \int_{G_{H}^{n-k-1}} \chi(\operatorname{Lk}(X \cap J)) d J d H=g_{2}^{1} \int_{G_{L}^{n-k-1}} \chi(\operatorname{Lk}(X \cap J)) d J,
$$


SO

$$
\mathcal{J}=\frac{g_{2}^{1}}{2 g_{n}^{n-k} g_{k}^{1} g_{n-k}^{n-k-1}} \int_{G_{n}^{n-k+1}} \int_{G_{L}^{n-k-1}} \chi(\operatorname{Lk}(X \cap J)) d J .
$$

To finish the computation, we consider the flag variety of pairs $(L, J), L \in$ $G_{n}^{n-k+1}$ and $J \in G_{L}^{n-k-1}$. It is a bundle over $G_{n}^{n-k-1}$, each fibre being a $G_{k+1}^{2}$. Hence we have

$$
\begin{gathered}
\mathcal{J}=\frac{g_{2}^{1}}{2 g_{n}^{n-k} g_{k}^{1} g_{n-k}^{n-k-1}} \int_{G_{n}^{n-k-1}} \int_{G_{J}^{2}} \chi(\operatorname{Lk}(X \cap J)) d J d M, \\
\mathcal{J}=\frac{g_{2}^{1} g_{k+1}^{2}}{2 g_{n}^{n-k} g_{k}^{1} g_{n-k}^{n-k-1}} \int_{G_{n}^{n-k-1}} \chi(\operatorname{Lk}(X \cap J)) d J= \\
\frac{1}{2 g_{n}^{n-k-1}} \int_{G_{n}^{n-k-1}} \chi(\operatorname{Lk}(X \cap J)) d J .
\end{gathered}
$$

This ends the proof for the case $k \in\{1, \ldots, n-2\}$. For $k=n-1$ or $n$, the proof is the same. We just have to remark that in these cases

$$
\beta_{0}(H, v)+\beta_{0}(H,-v)=\chi\left(\operatorname{Lk}\left(X \cap L_{v}\right)\right),
$$

and if $k=n-1, \operatorname{dim} L_{v}=2$ and if $k=n, \operatorname{dim} L_{v}=1$.

Let us end with some remarks on the $\operatorname{limits}_{\lim } \lim _{\epsilon \rightarrow 0} \frac{\Lambda_{k}\left(X, X \cap B_{\epsilon}\right)}{b_{k} \epsilon^{k}}$. We already know that if $\operatorname{dim} X=d$ then $\lim _{\epsilon \rightarrow 0} \frac{\Lambda_{k}\left(X, X \cap B_{\epsilon}\right)}{b_{k} \epsilon^{k}}=0$ for $k \geq d+1$. This is also the case if $l<d_{0}$, where $d_{0}$ is the dimension of the stratum that contains 0 . To see this let us first relate the limits $\lim _{\epsilon \rightarrow 0} \frac{\Lambda_{k}\left(X, X \cap B_{\epsilon}\right)}{b_{k} \epsilon^{k}}$ to the polar invariants defined by Comte and Merle in [9]. They can be defined as follows. Let $H \in G_{n}^{n-k}, k \in\{1, \ldots, n\}$, and let $v$ be an element in $S_{H^{\perp}}^{k-1}$. For $\delta>0$, we set

$$
\lambda_{0}(H, v)=\lim _{\epsilon \rightarrow 0} \lim _{\delta \rightarrow 0} \chi\left(H_{\delta, v} \cap X \cap B_{\epsilon}\right),
$$

and then

$$
\sigma_{k}(X, 0)=\frac{1}{s_{k-1}} \int_{S_{H^{\perp}}^{k-1}} \lambda_{0}(H, v) d v .
$$

Moreover, we put $\sigma_{0}(X, 0)=1$.

Theorem 5.6. For $k \in\{0, \ldots, n-1\}$, we have

$$
\lim _{\epsilon \rightarrow 0} \frac{\Lambda_{k}\left(X, X \cap B_{\epsilon}\right)}{b_{k} \epsilon^{k}}=\sigma_{k}(X, 0)-\sigma_{k+1}(X, 0) .
$$

Furthermore, we have

$$
\lim _{\epsilon \rightarrow 0} \frac{\Lambda_{n}\left(X, X \cap B_{\epsilon}\right)}{b_{n} \epsilon^{n}}=\sigma_{n}(X, 0) .
$$


Proof. It is the same proof as Theorem 5.5. For example if $k \in\{0, \ldots, n-1\}$, we just have to remark that

$$
\lambda_{0}(H, v)+\lambda_{0}(H,-v)=\chi\left(\operatorname{Lk}\left(X \cap L_{v}\right)\right)+\chi(\operatorname{Lk}(X \cap H)),
$$

by Lemma 5.2, which implies that

$\sigma_{k}(X, 0)=\frac{1}{2 g_{n}^{n-k+1}} \int_{G_{n}^{n-k+1}} \chi(\operatorname{Lk}(X \cap L)) d L+\frac{1}{2 g_{n}^{n-k}} \int_{G_{n}^{n-k}} \chi(\operatorname{Lk}(X \cap H)) d H$.

It is explained in [9] that $\sigma_{k}(X, 0)=1$ if $0 \leq k \leq d_{0}$, so if $k<d_{0}$ then $\lim _{\epsilon \rightarrow 0} \frac{\Lambda_{k}\left(X, X \cap B_{\epsilon}\right)}{b_{k} \epsilon^{k}}=0$.

\section{REFERENCES}

[1] AOKI, K., FUKUDA, T., NISHIMURA. T. : On the number of branches of the zero locus of a map germ $\left(\mathbf{R}^{n}, 0\right) \rightarrow\left(\mathbf{R}^{n-1}, 0\right)$. Topology and Computer Science: Proceedings of the Symposium held in honor of S. Kinoshita, H. Noguchi and T. Homma on the occasion of their sixtieth birhtdays (1987), 347-363.

[2] AOKI, K., FUKUDA, T., NISHIMURA. T. : An algebraic formula for the topological types of one parameter bifurcation diagrams, Archive for Rational Mechanics and Analysis 108 (1989), 247-265.

[3] AOKI, K., FUKUDA, T., SUN, W.Z. : On the number of branches of a plane curve germ, Kodai Math. Journal 9 (1986), 179-187.

[4] BEKKA, K.: Regular stratification of subanalytic sets, Bull. London Math. Soc. 25 no. 1 (1993), 7-16.

[5] BERNIG, A., BRÖCKER, L.: Courbures intrinsèques dans les catégories analytico-géométriques, Ann. Inst. Fourier (Grenoble) 53(6) (2003), 1897-1924.

[6] BRÖCKER, L., KUPPE, M.: Integral geometry of tame sets, Geometriae Dedicata 82 (2000), 285-323.

[7] CISNEROS-MOLINA, J. L., GRULHA JR., N. G., SEADE, J.: On the topology of real analytic maps, preprint (2012).

[8] COMTE, G.: Equisingularité réelle: nombres de Lelong et images polaires, Ann. Sci. Ecole Norm. Sup (4) 33(6) (2000), 757-788.

[9] COMTE, G., MERLE, M.: Equisingularité réelle II : invariants locaux et conditions de régularité, Ann. Sci. c. Norm. Supr. (4) 41 (2008), no. 2, 221-269.

[10] DURFEE, A.H.: Neighborhoods of algebraic sets, Trans. Am. Math. Soc. 276 (1983), no. $2,517-530$.

[11] DUTERTRE, N.: Degree formulas for a topological invariant of bifurcations of function germs, Kodai Math. J. 23, no. 3 (2000), 442-461.

[12] DUTERTRE, N.: On the Milnor fibre of a real map-germ, Hokkaido Mathematical Journal 31 (2002), 301-319.

[13] DUTERTRE, N.: On the Euler characteristics of real Milnor fibres of partially parallelizable maps of $\left(\mathbb{R}^{n}, 0\right) \rightarrow\left(\mathbb{R}^{2}, 0\right)$, Kodai Math. J. 32, no. 2 (2009), 324351.

[14] DUTERTRE, N. : Euler characteristic and Lipschitz-Killing curvatures of closed semi-algebraic sets, Geom. Dedicata 158, no.1 (2012),167-189.

[15] DUTERTRE, N. : On the topology of semi-algebraic functions on closed semialgebraic sets, Manuscripta Mathematica 139, no. 3-4 (2012), 415-441.

[16] EISENBUD, D., LEVINE, H.I. : An algebraic formula for the degree of a $C^{\infty}$ map-germ, Annals of Mathematics 106 (1977), 19-44. 
[17] FU, J.H.G. : Curvature measures of subanalytic sets, Amer. J. Math. 116 (1994), no. 4, 819-880.

[18] FUKUI, T. : An algebraic formula for a topological invariant of bifurcation of 1-parameter family of function-germs, in Stratifications, singularities, and differential equations, II (Marseille, 1990; Honolulu, HI, 1990), Travaux en cours 55, Hermann, Paris, 45-54 1997.

[19] FUKUI, T. : Mapping degree formula for 2-parameter bifurcation of functiongerms, Topology 32 (1993), 567-571.

[20] FUKUI, T., KHOVANSKII, A. : Mapping degree and Euler characteristic, Kodai Math. J. 29, no. 1 (2006), 144-162.

[21] GREUEL, G.M.: Der Gauss-Manin Zusammenhang isolierter Singularitäten von vollständingen Durschnitten, Math. Annalen 214 (1975), 235-266.

[22] LÊ, D.T.: Calcul du nombre de Milnor d'une singularité isolée d'intersection complète, Funct. Anal. Appl. 8 (1974), 45-52.

[23] SZAFRANIEC, Z. : On the number of branches of a 1-dimensional semi-analytic set, Kodai Math. Journal 11 (1988), 78-85.

Aix-Marseille Université, LATP, 39 rue F. Joliot-Curie, 13453 Marseille Cedex 13, France.

E-mail address: nicolas.dutertre@univ-amu.fr 